\title{
An Analysis of Hospital Costs for Childhood Cancer Care
}

\author{
Owen Tan, MComm¹; Deborah J. Schofield, $\mathrm{PhD}^{1}$; and Rupendra Shrestha, $\mathrm{PhD}^{1}$
}

\begin{abstract}
Background: This study used a linked dataset consisting of all childhood cancers recorded over the course of 10 years in New South Wales (NSW), Australia, to evaluate the hospital and emergency department costs (from a payer perspective) and resources used by patients with childhood cancer. We also analyzed determinants responsible for high-frequency hospital admissions, hospital length of stay (LoS), and hospital costs. Methods: We analyzed linked data at the individual patient level for a retrospective cohort of 2,966 patients with cancer aged $<18$ years with a diagnosis date between 2001 and 2012 from the NSW Central Cancer Registry, Australia. We reported costs and use of hospitalization and emergency department presentation 1 year before the date of diagnosis, 1 year after diagnosis, and 2 to 5 years after diagnosis. We also examined the association between cancer types and hospital admission and hospital costs from the payer perspective. Patient characteristics associated with the frequency of hospital admissions, hospital LoS, and hospital costs were also determined using a generalized linear model. Results: Most hospital admission costs occurred in the first year after diagnosis, accounting for $>70 \%$ of hospital costs within 5 years after diagnosis. The estimated median annual cost of hospitalization in the first year after diagnosis was $A \$ 88,964$ (interquartile range [IQR], $A \$ 34,399-A \$ 163,968$ ) for patients diagnosed at age 0 to 14 years and $A \$ 23,384$ (IQR, $A \$ 5,585-A \$ 91,565)$ for those diagnosed at age 15 to 17 years. Higher frequency of hospital admissions, hospital LoS, and hospital costs were significantly associated with younger age at cancer diagnosis, cancer metastases, and living in remote/disadvantaged socioeconomic areas. Conclusions: Our study represents one of the first in Australia to include detailed hospitalization cost information for all childhood cancer cases. This study highlights the high hospital use by pediatric patients and the importance of early diagnosis. Our findings also demonstrate the health inequities experienced by patients from remote areas and the lowest socioeconomic areas.
\end{abstract}

J Natl Compr Canc Netw 2022;20(2):126-135 doi: 10.6004/jnccn.2020.7802

${ }^{1}$ GenIMPACT: Centre for Economic Impacts of Genomic Medicine, Macquarie Business School, Macquarie University, Sydney, Australia.

\section{Background}

Childhood cancers have been reported to use a disproportionate amount of healthcare resources in many countries. ${ }^{1}$ The average cost of pediatric cancer hospitalization in 2009 was $\$ 40,400$ in the United States and was approximately 5 times higher than hospitalization for other pediatric conditions. ${ }^{2}$ In Canada, the hospitalization cost of treating childhood cancer in 2012 was reported to be as high as CAD $\$ 101,008$ per patient (aged 0-14 years), and the total direct healthcare cost, including hospitalization cost, for the same period was CAD $\$ 136,413 .^{3}$ The Australian Institute of Health and Welfare (AIHW) reported that $>80 \%$ of cancer costs in patients aged 0 to 14 years were attributable to their hospitalization costs. ${ }^{4}$ The 2013 AIHW report estimated total healthcare costs of cancer for children aged 0 to 14 years at $\mathrm{A} \$ 122$ million, with hospitalization costs alone being approximately $\mathrm{A} \$ 103$ million, in 2009. A recent report estimated that adolescent and young adult Australians diagnosed with cancer would incur a total cost of A $\$ 1.3$ million per person over their lifetimes, with hospital admission costs accounting for the largest component of health expenditure. ${ }^{5}$

However, previous studies of the hospitalization costs and use of childhood cancer care were often limited to certain types of cancer, had small sample sizes, or were dated. $^{6,7}$ Two recent studies conducted in Canadian settings developed a comprehensive costing method by linking cancer registry and healthcare administrative data. ${ }^{3,8}$ Similarly, our study represents a rigorous approach to evaluating the cost of disease via data linkage and is one of the first to report detailed hospital and emergency department (ED) cost information for all childhood cancer cases recorded over 10 years in New South Wales (NSW), Australia. The aims of this study were to estimate the hospital and ED resources used by children (aged $<18$ years) ${ }^{9,10}$ diagnosed with cancer and to estimate hospital and ED costs of childhood cancer within 5 years of diagnosis from a payer perspective. The study also identifies key variables that are associated with high frequency of hospital admissions, hospital length of stay (LoS), and cost of hospital admissions.

See JNCCN.org for supplemental online content. 


\section{Methods}

\section{Data Source}

The NSW Central Cancer Registry (CCR) contains records of people with cancer in NSW. ${ }^{11}$ The study cohort comprised 2,966 patients aged $<18$ years who were registered in the NSW CCR ${ }^{11}$ as being diagnosed with cancer between July 1, 2001, and December 31, 2012, which was the most recent date for which the data were available in the NSW CCR at the time of this analysis.

Each individual in the NSW CCR dataset was assigned a unique identifier by the Centre for Health Record Linkage, ${ }^{12}$ and the data for these individuals were extracted from other datasets, including the NSW Registry of Births, Deaths \& Marriages; NSW Cause of Death Unit Record File; NSW Admitted Patient Data Collection (APDC); and NSW Emergency Department Data Collection (see supplemental eTable 1, available with this article at JNCCN.org). The records were then linked based on the unique identifier for each patient.

\section{Hospital Inpatient and ED Service Use}

We analyzed the annual hospital LoS, annual number of emergency presentations, annual number of hospital admissions, cost of inpatient care, and cost of ED presentation by cancer type (Table $1^{13}$ ). Annual hospital LoS was estimated by summing the LoS for each admission in a year.

\section{Hospital and ED Costs}

Hospital treatment costs were estimated using Australian Refined Diagnosis Related Groups (AR-DRGs) and episode of care LoS. Cost estimates were obtained from the National Hospital Cost Data Collection estimates published by the Independent Hospital Pricing Authority for patients in public hospitals. ${ }^{14}$ For those in private hospitals, we used the Hospital Casemix Protocol average cost estimates for each AR-DRG. ${ }^{15}$ AR-DRGs is a system used to classify patient hospital admissions by connecting the number and type of patients treated in a hospital (known as hospital case mix) to the resources required by the hospital. Costs of ED presentation were estimated using the urgency-related group code from the NSW Emergency Department Data Collection. ${ }^{14}$

Based on data from the death registry, if a patient did not die within our analysis period, $\mathrm{A} \$ 0$ cost was assigned for the period when no hospital or ED visits occurred. If a patient died within our analysis period, we limited the analysis of hospital and ED data for the individual up to the time of death. All costs were reported from a payer's perspective. Costs reported were inflated to 2018 using the consumer price index published by the Australian Bureau of Statistics (ABS) ${ }^{16}$

\section{Geographic Location and Socioeconomic Status}

The geographic distribution of patients was assessed using the Accessibility/Remoteness Index of Australia (ARIA+), which was used by the ABS for the Remoteness Area classification. ${ }^{17}$ Accordingly, our cohort was categorized by reported geographical area into 4 groups: major cities, inner regional, outer regional, and remote and very remote.

Patients' socioeconomic status was assessed using the Index of Relative Socio-economic Disadvantage (IRSD), one of the Socio-Economic Indexes for Areas (SEIFA) developed by the ABS to rank areas in Australia according to their relative socioeconomic advantage and disadvantage.$^{18}$ The SEIFA IRSD measures relative disadvantage in specific geographical areas by assessing the proportion of the census population across multiple socioeconomic indicators. The SEIFA IRSD was then categorized into quintiles, with 1 being the least disadvantaged area and 5 being the most disadvantaged area.

Highest Degree of Spread Within 4 Months of Diagnosis The highest degree of spread within 4 months of diagnosis estimated the extent of the spread of cancer from its point of origin within 4 months of the cancer diagnosis and was based on information collected from a combination of pathology reports, inpatient notifications, and other treatment facilities. The variable was categorized as localized to tissue of origin, regional spread, adjacent organs and/or regional lymph nodes, distant metastases, and hematologic cancer with undefined cancer spread.

\section{Case Identification}

Using date of diagnosis recorded in the NSW CCR, we categorized hospital inpatient records and ED presentations into 1 year before the date of diagnosis, within 1 year of the date of diagnosis, and within 2 to 5 years of the date of diagnosis (reported as an annual average over 4 years). Analysis for people in years 2 through 5 excluded those who died within 1 year of diagnosis.

Admissions with a cancer-related ICD-10, Australian Modification (ICD-10-AM) code ${ }^{19}$ or a cancer-related morphology $\operatorname{code}^{20}$ as either the primary diagnosis or one of the secondary diagnoses were identified as cancerrelated hospital admissions. Using cancer-related diagnosis codes to identify hospital admissions relevant to the disease was demonstrated to be an effective classification method in other studies. ${ }^{21,22}$

We did not use ICD-10-AM codes to identify cancerrelated ED presentations. The rationale for this approach was that, because of the heterogeneity in ED diagnosis coding, patients presenting at the ED often were not recorded with an ICD-10-AM cancer diagnosis code. All presentations within 5 years of the date of cancer diagnosis were considered in our analysis. Because of limited data 


\begin{tabular}{|c|c|}
\hline Characteristic & n (\%) \\
\hline \multicolumn{2}{|l|}{ Sex } \\
\hline Male & $1,639(55.26)$ \\
\hline Female & $1,327(44.74)$ \\
\hline \multicolumn{2}{|l|}{ Age at diagnosis, y } \\
\hline $0-4$ & $1,117(37.66)$ \\
\hline $5-9$ & $528(17.80)$ \\
\hline $10-14$ & $632(21.31)$ \\
\hline $15-17$ & $689(23.23)$ \\
\hline \multicolumn{2}{|l|}{ Number of primary cancer sites } \\
\hline 1 & $2,924(97.66)$ \\
\hline$\geq 2$ & $70(2.34)$ \\
\hline \multicolumn{2}{|l|}{ Highest degree of spread within 4 mo of diagnosis } \\
\hline Localized to tissue of origin & $867(28.96)$ \\
\hline $\begin{array}{l}\text { Regional spread, invasion of adjacent organs and/or } \\
\text { regional lymph nodes }\end{array}$ & $264(8.82)$ \\
\hline Distant metastases & $231(7.72)$ \\
\hline Hematologic cancer with undefined cancer spread & $1,450(48.43)$ \\
\hline Nonhematologic cancer with unknown cancer spread & $182(6.08)$ \\
\hline \multicolumn{2}{|l|}{ SEIFA } \\
\hline Quintile 1 (least disadvantaged) & $578(19.49)$ \\
\hline Quintile 2 & $583(19.67)$ \\
\hline Quintile 3 & $561(18.93)$ \\
\hline Quintile 4 & $603(20.33)$ \\
\hline Quintile 5 (most disadvantaged) & $640(21.57)$ \\
\hline \multicolumn{2}{|l|}{ ARIA } \\
\hline Major cities & $2,159(72.79)$ \\
\hline Inner regional & $602(20.30)$ \\
\hline Outer regional & $190(6.41)$ \\
\hline Remote and very remote & $15(0.51)$ \\
\hline
\end{tabular}

(continued)

availability, analysis of ED presentations was limited to people diagnosed after 2005.

\section{Reason for Hospital Admissions}

Patients' hospital admissions were also reported by Major Diagnostic Categories (MDC). In the NSW APDC dataset, principal diagnoses were reported in 23 mutually exclusive MDC. ${ }^{23}$ The diagnoses in each category are related to a single body system or etiology, roughly reflecting the specialty of care. Each category is partitioned according to whether a surgical procedure was performed. We subdivided the hospital admission records into cancer-related and non-cancer-related admissions as described in the preceding "Case Identification" subsection, and reported the distribution of principal diagnosis by MDC.
Table 1. NSW Central Cancer Registry Patient Characteristics (cont.)

\begin{tabular}{|c|c|}
\hline Characteristic & n (\%) \\
\hline \multicolumn{2}{|l|}{ Cancer types } \\
\hline Acute lymphoblastic leukemia & $712(23.78)$ \\
\hline Brain & $342(11.42)$ \\
\hline Hodgkin lymphoma & $210(7.01)$ \\
\hline Non-Hodgkin lymphoma & $186(6.21)$ \\
\hline Bone & $177(5.91)$ \\
\hline Acute myeloid leukemia & $168(5.61)$ \\
\hline Connective tissue, peripheral nerves & $148(4.94)$ \\
\hline Kidney & $134(4.48)$ \\
\hline Other endocrine glands & $113(3.77)$ \\
\hline Melanoma of skin & $106(3.54)$ \\
\hline Colon & $70(2.34)$ \\
\hline Thyroid & $68(2.27)$ \\
\hline Testis & $67(2.24)$ \\
\hline Central nervous system & $67(2.24)$ \\
\hline III-defined and unspecified site & $60(2.00)$ \\
\hline Eye & $59(1.97)$ \\
\hline Other lymphatic, hematopoietic & $49(1.64)$ \\
\hline Liver & $41(1.37)$ \\
\hline Ovary & $41(1.37)$ \\
\hline Other myeloid leukemia & $27(0.90)$ \\
\hline Other thoracic organs & $20(0.67)$ \\
\hline All other cancer types & $129(4.31)$ \\
\hline
\end{tabular}

Abbreviations: ARIA, Accessibility/Remoteness Index of Australia; NSW, New South Wales; SEIFA, Socio-Economic Indexes for Areas. Data from NSW Central Cancer Registry. ${ }^{13}$

Outcome Measured, Reporting Cancer Types, and Statistical Analysis

We reported summary statistics (mean with standard deviation and median with interquartile range [IQR]) for frequency of annual hospital admissions, ED presentations per person, number of years spent in the hospital system (between the first cancer-related admission and the last cancer-related separation), annual hospital LoS, annual costs of hospital admissions, and ED presentations by cancer types (described in Table 1; for relevant ICD-O-3 codes, see supplemental eTable 2). Cancer types with $<20$ cases in the NSW CCR were grouped into "all other cancer types" (detailed in supplemental eTable 3 ). Because there were substantial differences in cancer distribution between younger and older age of diagnosis, we have included results separated into 2 age groups (0-14 and 15-17 years).

Age at diagnosis, sex, and advanced-stage cancer at diagnosis were reported by previous studies to influence childhood cancer survival rate. ${ }^{24}$ Geographic distribution 


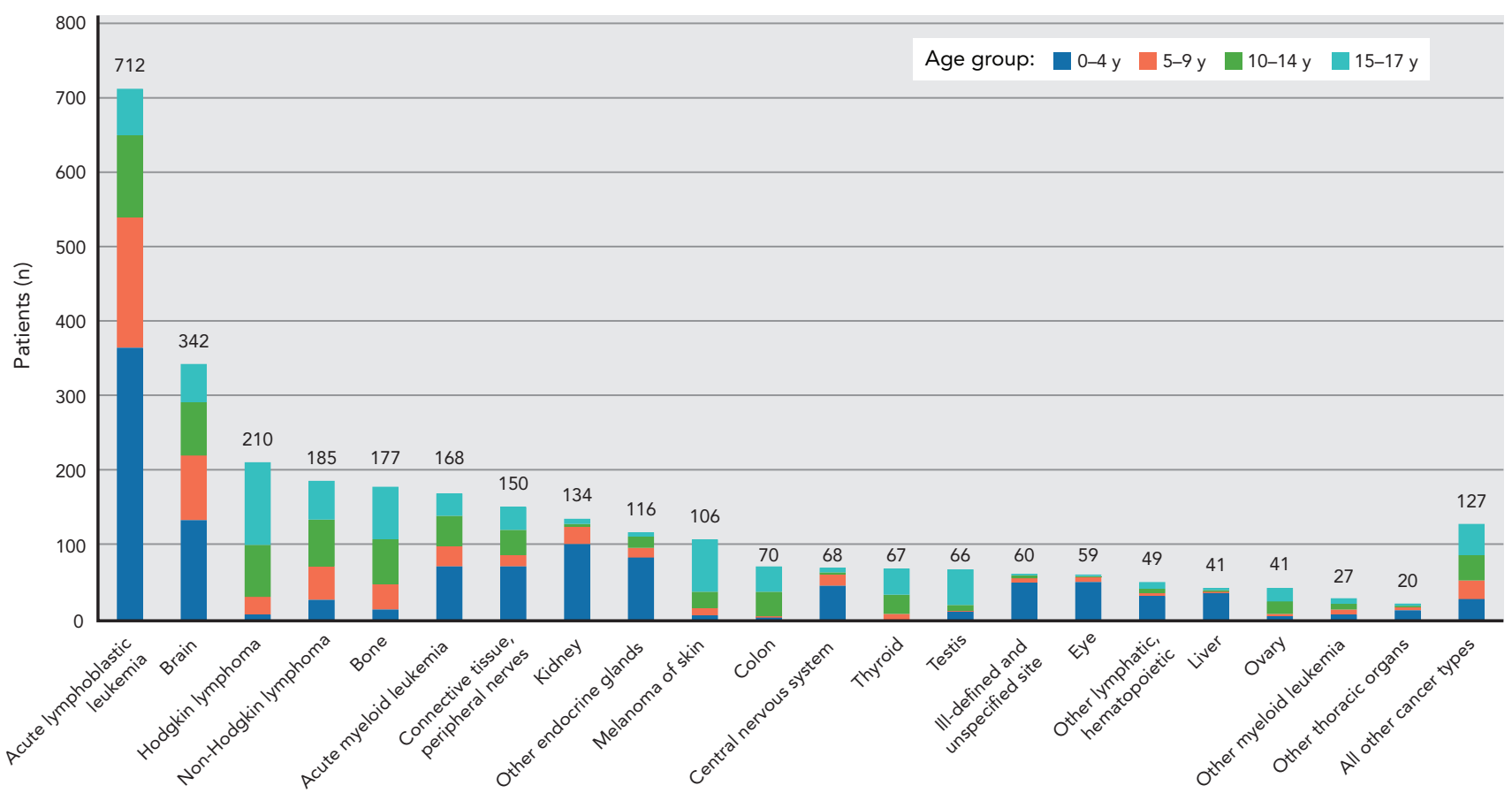

Figure 1. Distribution of cancer types recorded in NSW CCR (2001-2012) by age group.

and socioeconomic status were also reported to be associated with survival in adult cancers. ${ }^{25}$ Patient hospital stays were reported to be more prevalent in younger age groups. ${ }^{2}$ Drivers of the number of hospital admissions and hospital LoS in the first year after cancer diagnosis were analyzed using a generalized linear model (GLM) with a log-link negative binomial distribution with the following variables: age at diagnosis, sex, highest degree of spread within 4 months of diagnosis, cancer types, number of primary sites (as defined in the International Rules for Multiple Primary Cancers), ${ }^{26}$ ARIA+, SEIFA IRSD, and diagnosis year (2001-2012).

We used a similar modeling approach to analyze the drivers of hospital admission costs in the first year after diagnosis, using a GLM with a log-link gamma distribution with the same set of explanatory variables. All data analyses were performed using SAS 9.4 (SAS Institute Inc) and Microsoft Excel software.

\section{Results}

Among 2,966 patients with cancer (aged $<18$ years) in the NSW CCR, 55.3\% were male (Table 1), and 27 had $>1$ record in the cancer registry $(>1$ primary cancer as defined by the Cancer Institute NSW). ${ }^{13}$ Acute lymphoblastic leukemia (ALL), brain cancer, Hodgkin lymphoma, nonHodgkin lymphoma, and bone cancer together accounted for $55 \%$ of the reported cases (Figure 1). Among these cancers, ALL and brain cancer were more common among the younger age group ( $0-4$ years), whereas Hodgkin lymphoma and bone cancer were more common among the older age group (15-17 years). Non-Hodgkin lymphoma was most common in patients aged 10 to 14 years.

Most of the patients with cancer were from the major cities, closely aligning with the NSW population distribution by ARIA + . The childhood cancer cases were equally distributed among different socioeconomic groups, as represented by the SEIFA IRSD index (Table 1).

Most patients (97.7\%) had 1 primary cancer site, and $29 \%$ were diagnosed with localized cancers (Table 1).

\section{Hospital Admissions, ED Presentations, and Hospital LoS}

A total of 62,771 records of hospital admissions were included in the NSW APDC dataset. Of those, 54,399 records fell within 5 years of cancer diagnosis and 48,090 were cancer-related admissions. A total of 23,450 records of ED presentations were included in the NSW Emergency Department Data Collection dataset, and 16,401 occurred within 5 years of cancer diagnosis.

Most hospital admissions and ED presentations occurred in the first year after diagnosis, accounting for $71 \%$ of hospital admissions and $50 \%$ of ED presentations in the 5 years after diagnosis with cancer (ages 0-14 years) (Table 2, supplemental eTable 4). Patients with ALL had the highest number of hospital admissions within 5 years of being diagnosed with cancer ( 25 per person; IQR, 20-33) (Table 2). ALL (7 per person; IQR, 2-12) and other 


\section{Table 2. Annual Frequency of Hospital Admissions per Patient}

\begin{tabular}{|c|c|c|c|c|c|c|c|c|}
\hline \multirow[b]{2}{*}{ Parameter } & \multicolumn{2}{|c|}{1 y Before Diagnosis } & \multicolumn{2}{|c|}{1 y After Diagnosis } & \multicolumn{2}{|c|}{$\begin{array}{l}\text { 2-5 y After Diagnosis } \\
\text { (Annually) }\end{array}$} & \multicolumn{2}{|c|}{$\begin{array}{l}\text { Total of Years 1-5 } \\
\text { (Sum Over } 5 \text { y) }\end{array}$} \\
\hline & $\begin{array}{l}\text { Median } \\
\text { (IQR) }\end{array}$ & $\begin{array}{l}\text { Average } \\
\text { (SD) }\end{array}$ & $\begin{array}{l}\text { Median } \\
\text { (IQR) }\end{array}$ & $\begin{array}{l}\text { Average } \\
\text { (SD) }\end{array}$ & $\begin{array}{l}\text { Median } \\
\text { (IQR) }\end{array}$ & $\begin{array}{l}\text { Average } \\
\text { (SD) }\end{array}$ & $\begin{array}{l}\text { Median } \\
\text { (IOR) }\end{array}$ & $\begin{array}{l}\text { Average } \\
\text { (SD) }\end{array}$ \\
\hline \multicolumn{9}{|l|}{ Age at diagnosis } \\
\hline $0-14$ y & $\begin{array}{l}0.00 \\
(0.00-0.00)\end{array}$ & $\begin{array}{l}0.04 \\
(0.47)\end{array}$ & $\begin{array}{l}12.00 \\
(4.00-19.00)\end{array}$ & $\begin{array}{l}13.05 \\
(10.27)\end{array}$ & $\begin{array}{l}0.75 \\
(0.00-2.00)\end{array}$ & $\begin{array}{l}1.79 \\
(3.17)\end{array}$ & $\begin{array}{l}17.00 \\
(6.00-26.00)\end{array}$ & $\begin{array}{l}18.76 \\
(16.99)\end{array}$ \\
\hline $15-17 y$ & $\begin{array}{l}0.00 \\
(0.00-0.00)\end{array}$ & $\begin{array}{l}0.03 \\
(0.21)\end{array}$ & $\begin{array}{l}3.00 \\
(1.00-9.00)\end{array}$ & $\begin{array}{l}6.12 \\
(7.45)\end{array}$ & $\begin{array}{l}0.00 \\
(0.00-0.50)\end{array}$ & $\begin{array}{l}0.88 \\
(2.25)\end{array}$ & $\begin{array}{l}4.00 \\
(1.00-11.00)\end{array}$ & $\begin{array}{l}8.30 \\
(10.92)\end{array}$ \\
\hline \multicolumn{9}{|l|}{ By cancer type } \\
\hline $\begin{array}{l}\text { Acute lymphoblastic } \\
\text { leukemia }\end{array}$ & $\begin{array}{l}0.00 \\
(0.00-0.00)\end{array}$ & $\begin{array}{l}0.01 \\
(0.09)\end{array}$ & $\begin{array}{l}19.00 \\
(16.00-22.00)\end{array}$ & $\begin{array}{l}18.87 \\
(7.47)\end{array}$ & $\begin{array}{l}1.25 \\
(0.75-2.50)\end{array}$ & $\begin{array}{l}2.39 \\
(3.04)\end{array}$ & $\begin{array}{l}25.00 \\
(20.00-33.00)\end{array}$ & $\begin{array}{l}27.10 \\
(13.60)\end{array}$ \\
\hline Brain & $\begin{array}{l}0.00 \\
(0.00-0.00)\end{array}$ & $\begin{array}{l}0.06 \\
(0.30)\end{array}$ & $\begin{array}{l}6.00 \\
(2.00-13.00)\end{array}$ & $\begin{array}{l}8.79 \\
(9.91)\end{array}$ & $\begin{array}{l}0.75 \\
(0.00-2.25)\end{array}$ & $\begin{array}{l}1.65 \\
(2.51)\end{array}$ & $\begin{array}{l}8.00 \\
(2.00-17.00)\end{array}$ & $\begin{array}{l}12.62 \\
(14.06)\end{array}$ \\
\hline Hodgkin lymphoma & $\begin{array}{l}0.00 \\
(0.00-0.00)\end{array}$ & $\begin{array}{l}0.01 \\
(0.12)\end{array}$ & $\begin{array}{l}4.00 \\
(2.00-8.00)\end{array}$ & $\begin{array}{l}5.73 \\
(5.27)\end{array}$ & $\begin{array}{l}0.00 \\
(0.00-0.00)\end{array}$ & $\begin{array}{l}0.28 \\
(1.04)\end{array}$ & $\begin{array}{l}5.00 \\
(2.00-9.00)\end{array}$ & $\begin{array}{l}6.81 \\
(7.39)\end{array}$ \\
\hline $\begin{array}{l}\text { Non-Hodgkin } \\
\text { lymphoma }\end{array}$ & $\begin{array}{l}0.00 \\
(0.00-0.00)\end{array}$ & $\begin{array}{l}0.01 \\
(0.07)\end{array}$ & $\begin{array}{l}10.00 \\
(4.00-14.00)\end{array}$ & $\begin{array}{l}10.22 \\
(8.12)\end{array}$ & $\begin{array}{l}0.00 \\
(0.00-0.75)\end{array}$ & $\begin{array}{l}1.00 \\
(2.40)\end{array}$ & $\begin{array}{l}10.50 \\
(5.00-17.00)\end{array}$ & $\begin{array}{l}13.00 \\
(12.14)\end{array}$ \\
\hline Bone & $\begin{array}{l}0.00 \\
(0.00-0.00)\end{array}$ & $\begin{array}{l}0.02 \\
(0.18)\end{array}$ & $\begin{array}{l}15.00 \\
(6.00-22.00)\end{array}$ & $\begin{array}{l}14.63 \\
(9.98)\end{array}$ & $\begin{array}{l}0.50 \\
(0.00-2.50)\end{array}$ & $\begin{array}{l}1.85 \\
(3.03)\end{array}$ & $\begin{array}{l}20.00 \\
(7.00-28.00)\end{array}$ & $\begin{array}{l}19.58 \\
(14.31)\end{array}$ \\
\hline $\begin{array}{l}\text { Acute myeloid } \\
\text { leukemia }\end{array}$ & $\begin{array}{l}0.00 \\
(0.00-0.00)\end{array}$ & $\begin{array}{l}0.02 \\
(0.15)\end{array}$ & $\begin{array}{l}11.00 \\
(8.00-15.00)\end{array}$ & $\begin{array}{l}11.41 \\
(6.69)\end{array}$ & $\begin{array}{l}0.63 \\
(0.00-2.50)\end{array}$ & $\begin{array}{l}1.71 \\
(2.64)\end{array}$ & $\begin{array}{l}15.00 \\
(9.00-21.00)\end{array}$ & $\begin{array}{l}16.33 \\
(11.70)\end{array}$ \\
\hline $\begin{array}{l}\text { Connective tissue, } \\
\text { peripheral nerves }\end{array}$ & $\begin{array}{l}0.00 \\
(0.00-0.00)\end{array}$ & $\begin{array}{l}0.01 \\
(0.12)\end{array}$ & $\begin{array}{l}10.50 \\
(3.00-17.00)\end{array}$ & $\begin{array}{l}12.34 \\
(11.71)\end{array}$ & $\begin{array}{l}0.25 \\
(0.00-2.25)\end{array}$ & $\begin{array}{l}2.04 \\
(5.06)\end{array}$ & $\begin{array}{l}12.50 \\
(4.00-22.00)\end{array}$ & $\begin{array}{l}17.54 \\
(19.16)\end{array}$ \\
\hline Kidney & $\begin{array}{l}0.00 \\
(0.00-0.00)\end{array}$ & $\begin{array}{l}0.01 \\
(0.09)\end{array}$ & $\begin{array}{l}8.00 \\
(4.00-13.00)\end{array}$ & $\begin{array}{l}11.08 \\
(14.13)\end{array}$ & $\begin{array}{l}0.25 \\
(0.00-1.25)\end{array}$ & $\begin{array}{l}1.55 \\
(4.74)\end{array}$ & $\begin{array}{l}11.00 \\
(5.00-18.00)\end{array}$ & $\begin{array}{l}15.95 \\
(29.06)\end{array}$ \\
\hline $\begin{array}{l}\text { Other endocrine } \\
\text { glands }\end{array}$ & $\begin{array}{l}0.00 \\
(0.00-0.00)\end{array}$ & $\begin{array}{l}0.03 \\
(0.16)\end{array}$ & $\begin{array}{l}15.00 \\
(8.00-19.00)\end{array}$ & $\begin{array}{l}15.09 \\
(10.53)\end{array}$ & $\begin{array}{l}1.50 \\
(0.00-3.50)\end{array}$ & $\begin{array}{l}2.76 \\
(4.27)\end{array}$ & $\begin{array}{l}21.00 \\
(8.00-28.00)\end{array}$ & $\begin{array}{l}22.39 \\
(18.08)\end{array}$ \\
\hline Melanoma of skin & $\begin{array}{l}0.00 \\
(0.00-0.00)\end{array}$ & $\begin{array}{l}0.01 \\
(0.10)\end{array}$ & $\begin{array}{l}1.00 \\
(0.00-1.00)\end{array}$ & $\begin{array}{l}1.01 \\
(1.08)\end{array}$ & $\begin{array}{l}0.00 \\
(0.00-0.00)\end{array}$ & $\begin{array}{l}0.14 \\
(0.72)\end{array}$ & $\begin{array}{l}1.00 \\
(0.00-2.00)\end{array}$ & $\begin{array}{l}1.43 \\
(2.54)\end{array}$ \\
\hline Colon & $\begin{array}{l}0.00 \\
(0.00-0.00)\end{array}$ & $\begin{array}{l}0.07 \\
(0.60)\end{array}$ & $\begin{array}{l}1.00 \\
(0.00-1.00)\end{array}$ & $\begin{array}{l}1.04 \\
(1.00)\end{array}$ & $\begin{array}{l}0.00 \\
(0.00-0.00)\end{array}$ & $\begin{array}{l}0.07 \\
(0.49)\end{array}$ & $\begin{array}{l}1.00 \\
(0.00-1.00)\end{array}$ & $\begin{array}{l}1.13 \\
(1.15)\end{array}$ \\
\hline Thyroid & $\begin{array}{l}0.00 \\
(0.00-0.00)\end{array}$ & $\begin{array}{l}0.03 \\
(0.17)\end{array}$ & $\begin{array}{l}2.00 \\
(2.00-3.00)\end{array}$ & $\begin{array}{l}2.56 \\
(2.04)\end{array}$ & $\begin{array}{l}0.00 \\
(0.00-0.25)\end{array}$ & $\begin{array}{l}0.15 \\
(0.27)\end{array}$ & $\begin{array}{l}3.00 \\
(2.00-3.00)\end{array}$ & $\begin{array}{l}3.18 \\
(2.61)\end{array}$ \\
\hline Testis & $\begin{array}{l}0.00 \\
(0.00-0.00)\end{array}$ & $\begin{array}{l}0.01 \\
(0.12)\end{array}$ & $\begin{array}{l}3.00 \\
(1.00-9.00)\end{array}$ & $\begin{array}{l}6.93 \\
(8.72)\end{array}$ & $\begin{array}{l}0.00 \\
(0.00-0.25)\end{array}$ & $\begin{array}{l}0.62 \\
(2.03)\end{array}$ & $\begin{array}{l}4.00 \\
(1.00-14.00)\end{array}$ & $\begin{array}{l}8.42 \\
(10.14)\end{array}$ \\
\hline $\begin{array}{l}\text { Central nervous } \\
\text { system }\end{array}$ & $\begin{array}{l}0.00 \\
(0.00-0.00)\end{array}$ & $\begin{array}{l}0.52 \\
(2.25)\end{array}$ & $\begin{array}{l}3.00 \\
(1.00-7.00)\end{array}$ & $\begin{array}{l}5.39 \\
(6.05)\end{array}$ & $\begin{array}{l}1.25 \\
(0.25-2.75)\end{array}$ & $\begin{array}{l}1.95 \\
(2.40)\end{array}$ & $\begin{array}{l}9.50 \\
(3.00-18.00)\end{array}$ & $\begin{array}{l}12.52 \\
(14.03)\end{array}$ \\
\hline $\begin{array}{l}\text { Ill-defined and } \\
\text { unspecified site }\end{array}$ & $\begin{array}{l}0.00 \\
(0.00-0.00)\end{array}$ & $\begin{array}{l}0.03 \\
(0.26)\end{array}$ & $\begin{array}{l}7.50 \\
(2.00-17.50)\end{array}$ & $\begin{array}{l}11.18 \\
(10.54)\end{array}$ & $\begin{array}{l}0.50 \\
(0.00-2.50)\end{array}$ & $\begin{array}{l}1.76 \\
(2.49)\end{array}$ & $\begin{array}{l}13.50 \\
(3.00-24.50)\end{array}$ & $\begin{array}{l}17.17 \\
(16.41)\end{array}$ \\
\hline Eye & $\begin{array}{l}0.00 \\
(0.00-0.00)\end{array}$ & $\begin{array}{l}0.00 \\
(0.00)\end{array}$ & $\begin{array}{l}8.00 \\
(4.00-14.00)\end{array}$ & $\begin{array}{l}9.68 \\
(7.80)\end{array}$ & $\begin{array}{l}1.50 \\
(0.00-3.50)\end{array}$ & $\begin{array}{l}2.87 \\
(3.57)\end{array}$ & $\begin{array}{l}13.00 \\
(6.00-31.00)\end{array}$ & $\begin{array}{l}20.78 \\
(19.66)\end{array}$ \\
\hline $\begin{array}{l}\text { Other lymphatic, } \\
\text { hematopoietic }\end{array}$ & $\begin{array}{l}0.00 \\
(0.00-0.00)\end{array}$ & $\begin{array}{l}0.27 \\
(1.17)\end{array}$ & $\begin{array}{l}2.00 \\
(1.00-7.00)\end{array}$ & $\begin{array}{l}5.65 \\
(8.14)\end{array}$ & $\begin{array}{l}0.00 \\
(0.00-1.25)\end{array}$ & $\begin{array}{l}1.10 \\
(2.04)\end{array}$ & $\begin{array}{l}2.00 \\
(1.00-13.00)\end{array}$ & $\begin{array}{l}9.73 \\
(14.22)\end{array}$ \\
\hline Liver & $\begin{array}{l}0.00 \\
(0.00-0.00)\end{array}$ & $\begin{array}{l}0.02 \\
(0.16)\end{array}$ & $\begin{array}{l}13.50 \\
(8.50-17.50)\end{array}$ & $\begin{array}{l}13.68 \\
(8.43)\end{array}$ & $\begin{array}{l}0.25 \\
(0.00-1.25)\end{array}$ & $\begin{array}{l}0.89 \\
(1.97)\end{array}$ & $\begin{array}{l}14.00 \\
(9.00-20.50)\end{array}$ & $\begin{array}{l}16.53 \\
(12.18)\end{array}$ \\
\hline Ovary & $\begin{array}{l}0.00 \\
(0.00-0.00)\end{array}$ & $\begin{array}{l}0.00 \\
(0.00)\end{array}$ & $\begin{array}{l}5.00 \\
(1.00-8.00)\end{array}$ & $\begin{array}{l}5.66 \\
(5.13)\end{array}$ & $\begin{array}{l}0.00 \\
(0.00-0.00)\end{array}$ & $\begin{array}{l}0.08 \\
(0.33)\end{array}$ & $\begin{array}{l}5.00 \\
(1.00-8.00)\end{array}$ & $\begin{array}{l}5.88 \\
(5.29)\end{array}$ \\
\hline $\begin{array}{l}\text { Other myeloid } \\
\text { leukemia }\end{array}$ & $\begin{array}{l}0.00 \\
(0.00-0.00)\end{array}$ & $\begin{array}{l}0.04 \\
(0.19)\end{array}$ & $\begin{array}{l}4.00 \\
(1.00-5.00)\end{array}$ & $\begin{array}{l}3.74 \\
(2.84)\end{array}$ & $\begin{array}{l}0.25 \\
(0.00-1.00)\end{array}$ & $\begin{array}{l}0.75 \\
(1.38)\end{array}$ & $\begin{array}{l}4.00 \\
(1.00-8.00)\end{array}$ & $\begin{array}{l}6.19 \\
(7.22)\end{array}$ \\
\hline $\begin{array}{l}\text { Other thoracic } \\
\text { organs }\end{array}$ & $\begin{array}{l}0.00 \\
(0.00-0.00)\end{array}$ & $\begin{array}{l}0.00 \\
(0.00)\end{array}$ & $\begin{array}{l}12.00 \\
(6.50-24.00)\end{array}$ & $\begin{array}{l}15.00 \\
(10.05)\end{array}$ & $\begin{array}{l}0.88 \\
(0.25-3.00)\end{array}$ & $\begin{array}{l}2.94 \\
(4.05)\end{array}$ & $\begin{array}{l}19.50 \\
(10.50-30.00)\end{array}$ & $\begin{array}{l}24.80 \\
(21.81)\end{array}$ \\
\hline $\begin{array}{l}\text { All other cancer } \\
\text { types }\end{array}$ & $\begin{array}{l}0.00 \\
(0.00-0.00)\end{array}$ & $\begin{array}{l}0.05 \\
(0.46)\end{array}$ & $\begin{array}{l}4.00 \\
(1.00-12.50)\end{array}$ & $\begin{array}{l}9.25 \\
(11.61)\end{array}$ & $\begin{array}{l}0.00 \\
(0.00-1.00)\end{array}$ & $\begin{array}{l}1.09 \\
(2.37)\end{array}$ & $\begin{array}{l}6.00 \\
(1.00-19.00)\end{array}$ & $\begin{array}{l}12.84 \\
(15.81)\end{array}$ \\
\hline
\end{tabular}

Abbreviation: IQR, interquartile range. 
endocrine gland cancers (including malignancy of adrenal gland and other endocrine glands and related structures; 7 per person [IQR, 2-11]) had the highest number of ED presentations over the course of 5 years (supplemental eTable 4).

The median total hospital LoS values within 5 years of cancer diagnosis were 61 days (IQR, 21-109 days) for patients diagnosed with cancer at 0 to 14 years of age and 14 days (IQR, 2-63 days) for those diagnosed at 15 to 17 years of age (Table 3). Patients with acute myeloid leukemia (AML) spent the most days in the hospital, with a median of 129.5 days (IQR, 83-182.5 days) in the 5-year period after diagnosis. The estimated average LoS per admission was 4.4 days (supplemental eTable 5).

The median number of years spent in the hospital system (between the first cancer-related admission and the last cancer-related separation) was 1.84 (IQR, 0.63-2.49) for patients diagnosed at 0 to 14 years of age, and 0.48 (IQR, 0-1.37) for those diagnosed at 15 to 17 years of age (supplemental eTable 6). Patients with cancer of the central nervous system had the longest time in the hospital system, with a median of 3.35 years (IQR, 0.59-4.37 years).

Costs of Hospital Admissions and ED Presentations Most hospital admission costs occurred in the first year after diagnosis, accounting for $>70 \%$ of hospital costs within 5 years after diagnosis (Table 4). The estimated median annual cost of hospitalization in the first year after diagnosis was A $\$ 88,964$ (IQR, A $\$ 34,399-A \$ 163,968$ ) for patients diagnosed at 0 to 14 years of age, and $A \$ 23,834$ (IQR, A\$5,585-A \$91,565) for those diagnosed at 15 to 17 years of age (Table 4 ). The median cost of ED presentations was $\mathrm{A} \$ 1,782$ (IQR, $\mathrm{A} \$ 0-\mathrm{A} \$ 4,902$ ) per patient in the first year after diagnosis for patients diagnosed at 0 to 14 years of age (supplemental eTable 7).

Patients with AML had the highest cost of hospitalization per patient in the 5 years after diagnosis, at a cost of A $\$ 239,218$ (IQR, A $\$ 140,434-A \$ 358,797$ ) (Table 4). Patients with ALL had the highest ED costs per patient in the 5 years after diagnosis, at $\mathrm{A} \$ 5,467$ (IQR, A $\$ 1,854-\mathrm{A} \$ 10,206$ ) per patient (supplemental eTable 7). Total costs of all cancerrelated hospital admissions and ED presentations were approximately A $\$ 448$ million over the 5 -year period after cancer diagnosis for all patients in NSW during 2001 through 2012 (supplemental eTable 8).

\section{Reason for Hospital Admission, by MDC}

In the cancer-related hospital admission dataset, a substantial proportion (44\%) of the admissions fell into the "neoplastic disorders" MDC (supplemental eTable 9). In the non-cancer-related hospital admission dataset, most admissions fell into the "factors influencing health status and other contacts with health services" MDC (supplemental eTable 9). Some of the common ICD-10AM codes related to this MDC in the dataset were Z45 (adjustment and management of drug delivery or implanted device) and Z50 (care involving use of rehabilitation procedures).

\section{Drivers for Hospital Admissions and Costs in Year 1 After Diagnosis}

Because most hospital admissions occurred in the first year after diagnosis, we analyzed drivers for year 1 hospital admissions, LoS, and costs by using a GLM. A higher frequency of hospital admissions in the first year after diagnosis was significantly associated with being male, younger age at cancer diagnosis, having highest cancer spread (regional or distant metastases) within 4 months of diagnosis, and living in a remote area (supplemental eTable 10). Children diagnosed with ALL were admitted more frequently than those with other cancers in the first year after diagnosis. We observed that patients living in the second least disadvantaged SEIFA IRSD and those living in the most disadvantaged SEIFA IRSD had fewer hospital admissions than those living in the least disadvantaged SEIFA IRSD.

Similarly, these variables were analyzed as predictors of hospital costs and hospital LoS. Higher hospital costs and longer hospital LoS were significantly associated with cancer diagnosis at a younger age, highest cancer spread (regional or distant metastases) within 4 months of diagnosis, and living in the most disadvantaged of the SEIFA IRSD areas (supplemental eTable 10). Having been diagnosed with either brain or bone cancer resulted in $>2$ times higher hospital costs than having been diagnosed with ALL (reference cancer type).

\section{Discussion}

Our study reported hospital and ED cost information for all childhood cancers diagnosed in NSW, Australia, from 2001 through 2012. Hospital and ED costs and use for many cancer types in our study have not been reported previously in Australia.

Using linked data from multiple data sources, this article provides robust estimates of the hospital resources used for treating childhood cancer in Australia. Analyzing linked data from multiple registries has been shown to be an improved method of estimating healthcare costs ${ }^{3,8,27}$ when compared with studies with small sample sizes or a limited observation period.

Our findings show that childhood cancer, although rare in occurrence, required extensive hospital system support, and for specific types of childhood cancer, such as AML, the total hospital LoS can be as long as 100 days in the first year after diagnosis. The average cost of hospital admission for all patients with cancer in the first year after diagnosis was significantly higher than the national 
Table 3. Length of Hospital Stay, in Days, per Patient

\begin{tabular}{|c|c|c|c|c|c|c|c|c|}
\hline \multirow[b]{2}{*}{ Parameter } & \multicolumn{2}{|c|}{1 y Before Diagnosis } & \multicolumn{2}{|c|}{1 y After Diagnosis } & \multicolumn{2}{|c|}{$\begin{array}{l}\text { 2-5 y After Diagnosis } \\
\text { (Annually) }\end{array}$} & \multicolumn{2}{|c|}{$\begin{array}{c}\text { Total of Years 1-5 } \\
\text { (Sum Over } 5 \text { y) }\end{array}$} \\
\hline & $\begin{array}{l}\text { Median } \\
\text { (IQR) }\end{array}$ & $\begin{array}{l}\text { Average } \\
\text { (SD) }\end{array}$ & $\begin{array}{l}\text { Median } \\
\text { (IQR) }\end{array}$ & $\begin{array}{l}\text { Average } \\
\text { (SD) }\end{array}$ & $\begin{array}{l}\text { Median } \\
\text { (IQR) }\end{array}$ & $\begin{array}{l}\text { Average } \\
\text { (SD) }\end{array}$ & $\begin{array}{l}\text { Median } \\
\text { (IQR) }\end{array}$ & $\begin{array}{l}\text { Average } \\
\text { (SD) }\end{array}$ \\
\hline \multicolumn{9}{|l|}{ Age at diagnosis } \\
\hline $0-14$ y & $\begin{array}{l}0.00 \\
(0.00-0.00)\end{array}$ & $\begin{array}{l}0.16 \\
(3.68)\end{array}$ & $\begin{array}{l}51.00 \\
(16.00-84.00)\end{array}$ & $\begin{array}{l}58.61 \\
(51.16)\end{array}$ & $\begin{array}{l}1.00 \\
(0.00-4.75)\end{array}$ & $\begin{array}{l}8.05 \\
(19.32)\end{array}$ & $\begin{array}{l}61.00 \\
(21.00-109.00)\end{array}$ & $\begin{array}{l}80.31 \\
(78.39)\end{array}$ \\
\hline $15-17 y$ & $\begin{array}{l}0.00 \\
(0.00-0.00)\end{array}$ & $\begin{array}{l}0.12 \\
(1.78)\end{array}$ & $\begin{array}{l}11.00 \\
(2.00-50.00)\end{array}$ & $\begin{array}{l}31.28 \\
(42.72)\end{array}$ & $\begin{array}{l}0.00 \\
(0.00-1.00)\end{array}$ & $\begin{array}{l}6.20 \\
(18.14)\end{array}$ & $\begin{array}{l}14.00 \\
(2.00-63.00)\end{array}$ & $\begin{array}{l}44.55 \\
(63.56)\end{array}$ \\
\hline \multicolumn{9}{|l|}{ By cancer type } \\
\hline $\begin{array}{l}\text { Acute lymphoblastic } \\
\text { leukemia }\end{array}$ & $\begin{array}{l}0.00 \\
(0.00-0.00)\end{array}$ & $\begin{array}{l}0.01 \\
(0.19)\end{array}$ & $\begin{array}{l}63.00 \\
(48.00-86.00)\end{array}$ & $\begin{array}{l}72.13 \\
(40.19)\end{array}$ & $\begin{array}{l}2.50 \\
(1.00-7.50)\end{array}$ & $\begin{array}{l}12.99 \\
(26.03)\end{array}$ & $\begin{array}{l}78.00 \\
(58.00-136.00)\end{array}$ & $\begin{array}{l}109.72 \\
(82.30)\end{array}$ \\
\hline Brain & $\begin{array}{l}0.00 \\
(0.00-0.00)\end{array}$ & $\begin{array}{l}0.18 \\
(1.18)\end{array}$ & $\begin{array}{l}32.00 \\
(8.00-74.00)\end{array}$ & $\begin{array}{l}48.12 \\
(48.81)\end{array}$ & $\begin{array}{l}1.00 \\
(0.00-6.75)\end{array}$ & $\begin{array}{l}7.58 \\
(16.91)\end{array}$ & $\begin{array}{l}43.00 \\
(13.00-85.00)\end{array}$ & $\begin{array}{l}61.68 \\
(65.15)\end{array}$ \\
\hline Hodgkin lymphoma & $\begin{array}{l}0.00 \\
(0.00-0.00)\end{array}$ & $\begin{array}{l}0.01 \\
(0.12)\end{array}$ & $\begin{array}{l}12.00 \\
(4.00-22.00)\end{array}$ & $\begin{array}{l}15.80 \\
(16.36)\end{array}$ & $\begin{array}{l}0.00 \\
(0.00-0.00)\end{array}$ & $\begin{array}{l}1.05 \\
(3.79)\end{array}$ & $\begin{array}{l}13.00 \\
(4.00-25.00)\end{array}$ & $\begin{array}{l}19.77 \\
(24.05)\end{array}$ \\
\hline $\begin{array}{l}\text { Non-Hodgkin } \\
\text { lymphoma }\end{array}$ & $\begin{array}{l}0.00 \\
(0.00-0.00)\end{array}$ & $\begin{array}{l}0.01 \\
(0.07)\end{array}$ & $\begin{array}{l}54.00 \\
(25.00-74.00)\end{array}$ & $\begin{array}{l}57.69 \\
(44.21)\end{array}$ & $\begin{array}{l}0.00 \\
(0.00-1.00)\end{array}$ & $\begin{array}{l}6.10 \\
(18.90)\end{array}$ & $\begin{array}{l}59.00 \\
(29.00-90.00)\end{array}$ & $\begin{array}{l}69.58 \\
(61.30)\end{array}$ \\
\hline Bone & $\begin{array}{l}0.00 \\
(0.00-0.00)\end{array}$ & $\begin{array}{l}0.03 \\
(0.25)\end{array}$ & $\begin{array}{l}73.00 \\
(22.00-100.00)\end{array}$ & $\begin{array}{l}68.27 \\
(47.84)\end{array}$ & $\begin{array}{l}1.25 \\
(0.00-8.75)\end{array}$ & $\begin{array}{l}8.88 \\
(15.08)\end{array}$ & $\begin{array}{l}86.00 \\
(35.00-128.00)\end{array}$ & $\begin{array}{l}90.82 \\
(69.59)\end{array}$ \\
\hline $\begin{array}{l}\text { Acute myeloid } \\
\text { leukemia }\end{array}$ & $\begin{array}{l}0.00 \\
(0.00-0.00)\end{array}$ & $\begin{array}{l}0.20 \\
(2.11)\end{array}$ & $\begin{array}{l}100.50 \\
(70.00-139.50)\end{array}$ & $\begin{array}{l}102.51 \\
(53.05)\end{array}$ & $\begin{array}{l}1.00 \\
(0.00-20.75)\end{array}$ & $\begin{array}{l}14.39 \\
(28.71)\end{array}$ & $\begin{array}{l}129.50 \\
(83.00-182.50)\end{array}$ & $\begin{array}{l}136.17 \\
(79.45)\end{array}$ \\
\hline $\begin{array}{l}\text { Connective tissue, } \\
\text { peripheral nerves }\end{array}$ & $\begin{array}{l}0.00 \\
(0.00-0.00)\end{array}$ & $\begin{array}{l}0.05 \\
(0.50)\end{array}$ & $\begin{array}{l}36.50 \\
(14.00-73.00)\end{array}$ & $\begin{array}{l}49.78 \\
(46.84)\end{array}$ & $\begin{array}{l}0.25 \\
(0.00-5.00)\end{array}$ & $\begin{array}{l}7.99 \\
(18.34)\end{array}$ & $\begin{array}{l}42.50 \\
(15.00-89.00)\end{array}$ & $\begin{array}{l}66.34 \\
(70.65)\end{array}$ \\
\hline Kidney & $\begin{array}{l}0.00 \\
(0.00-0.00)\end{array}$ & $\begin{array}{l}0.02 \\
(0.26)\end{array}$ & $\begin{array}{l}26.00 \\
(18.00-57.00)\end{array}$ & $\begin{array}{l}44.77 \\
(65.33)\end{array}$ & $\begin{array}{l}0.25 \\
(0.00-2.00)\end{array}$ & $\begin{array}{l}4.75 \\
(11.35)\end{array}$ & $\begin{array}{l}35.50 \\
(19.00-68.00)\end{array}$ & $\begin{array}{l}56.96 \\
(76.72)\end{array}$ \\
\hline $\begin{array}{l}\text { Other endocrine } \\
\text { glands }\end{array}$ & $\begin{array}{l}0.00 \\
(0.00-0.00)\end{array}$ & $\begin{array}{l}0.04 \\
(0.23)\end{array}$ & $\begin{array}{l}88.00 \\
(32.00-142.00)\end{array}$ & $\begin{array}{l}92.61 \\
(67.44)\end{array}$ & $\begin{array}{l}2.25 \\
(0.00-10.00)\end{array}$ & $\begin{array}{l}8.85 \\
(16.96)\end{array}$ & $\begin{array}{l}106.00 \\
(38.00-177.00)\end{array}$ & $\begin{array}{l}113.57 \\
(82.93)\end{array}$ \\
\hline Melanoma of skin & $\begin{array}{l}0.00 \\
(0.00-0.00)\end{array}$ & $\begin{array}{l}0.01 \\
(0.10)\end{array}$ & $\begin{array}{l}1.00 \\
(0.00-2.00)\end{array}$ & $\begin{array}{l}2.08 \\
(4.45)\end{array}$ & $\begin{array}{l}0.00 \\
(0.00-0.00)\end{array}$ & $\begin{array}{l}0.94 \\
(7.76)\end{array}$ & $\begin{array}{l}1.00 \\
(0.00-2.00)\end{array}$ & $\begin{array}{l}3.58 \\
(10.40)\end{array}$ \\
\hline Colon & $\begin{array}{l}0.00 \\
(0.00-0.00)\end{array}$ & $\begin{array}{l}2.43 \\
(20.32)\end{array}$ & $\begin{array}{l}2.00 \\
(0.00-6.00)\end{array}$ & $\begin{array}{l}6.11 \\
(11.93)\end{array}$ & $\begin{array}{l}0.00 \\
(0.00-0.00)\end{array}$ & $\begin{array}{l}1.02 \\
(8.01)\end{array}$ & $\begin{array}{l}2.00 \\
(0.00-6.00)\end{array}$ & $\begin{array}{l}7.26 \\
(15.46)\end{array}$ \\
\hline Thyroid & $\begin{array}{l}0.00 \\
(0.00-0.00)\end{array}$ & $\begin{array}{l}0.03 \\
(0.17)\end{array}$ & $\begin{array}{l}5.00 \\
(3.00-7.00)\end{array}$ & $\begin{array}{l}7.22 \\
(11.86)\end{array}$ & $\begin{array}{l}0.00 \\
(0.00-0.75)\end{array}$ & $\begin{array}{l}0.43 \\
(0.75)\end{array}$ & $\begin{array}{l}5.50 \\
(4.00-9.50)\end{array}$ & $\begin{array}{l}8.94 \\
(13.57)\end{array}$ \\
\hline Testis & $\begin{array}{l}0.00 \\
(0.00-0.00)\end{array}$ & $\begin{array}{l}0.01 \\
(0.12)\end{array}$ & $\begin{array}{l}9.00 \\
(1.00-32.00)\end{array}$ & $\begin{array}{l}21.94 \\
(32.23)\end{array}$ & $\begin{array}{l}0.00 \\
(0.00-0.25)\end{array}$ & $\begin{array}{l}2.18 \\
(9.54)\end{array}$ & $\begin{array}{l}14.00 \\
(1.00-38.00)\end{array}$ & $\begin{array}{l}26.13 \\
(35.67)\end{array}$ \\
\hline $\begin{array}{l}\text { Central nervous } \\
\text { system }\end{array}$ & $\begin{array}{l}0.00 \\
(0.00-0.00)\end{array}$ & $\begin{array}{l}0.66 \\
(2.70)\end{array}$ & $\begin{array}{l}8.00 \\
(2.00-17.00)\end{array}$ & $\begin{array}{l}17.95 \\
(31.10)\end{array}$ & $\begin{array}{l}2.50 \\
(0.25-4.75)\end{array}$ & $\begin{array}{l}4.36 \\
(6.72)\end{array}$ & $\begin{array}{l}19.50 \\
(6.00-36.00)\end{array}$ & $\begin{array}{l}32.61 \\
(43.03)\end{array}$ \\
\hline $\begin{array}{l}\text { Ill-defined and } \\
\text { unspecified site }\end{array}$ & $\begin{array}{l}0.00 \\
(0.00-0.00)\end{array}$ & $\begin{array}{l}0.15 \\
(1.16)\end{array}$ & $\begin{array}{l}40.50 \\
(15.50-102.00)\end{array}$ & $\begin{array}{l}59.10 \\
(52.07)\end{array}$ & $\begin{array}{l}0.50 \\
(0.00-8.75)\end{array}$ & $\begin{array}{l}8.03 \\
(14.98)\end{array}$ & $\begin{array}{l}56.50 \\
(21.50-128.00)\end{array}$ & $\begin{array}{l}83.30 \\
(83.43)\end{array}$ \\
\hline Eye & $\begin{array}{l}0.00 \\
(0.00-0.00)\end{array}$ & $\begin{array}{l}0.00 \\
(0.00)\end{array}$ & $\begin{array}{l}13.00 \\
(7.00-27.00)\end{array}$ & $\begin{array}{l}17.37 \\
(15.11)\end{array}$ & $\begin{array}{l}1.50 \\
(0.00-5.25)\end{array}$ & $\begin{array}{l}3.90 \\
(6.38)\end{array}$ & $\begin{array}{l}18.00 \\
(8.00-45.00)\end{array}$ & $\begin{array}{l}32.07 \\
(33.35)\end{array}$ \\
\hline $\begin{array}{l}\text { Other lymphatic, } \\
\text { hematopoietic }\end{array}$ & $\begin{array}{l}0.00 \\
(0.00-0.00)\end{array}$ & $\begin{array}{l}1.31 \\
(6.66)\end{array}$ & $\begin{array}{l}8.00 \\
(1.00-21.00)\end{array}$ & $\begin{array}{l}23.47 \\
(37.43)\end{array}$ & $\begin{array}{l}0.00 \\
(0.00-1.50)\end{array}$ & $\begin{array}{l}4.50 \\
(11.15)\end{array}$ & $\begin{array}{l}12.00 \\
(2.00-33.00)\end{array}$ & $\begin{array}{l}40.47 \\
(68.54)\end{array}$ \\
\hline Liver & $\begin{array}{l}0.00 \\
(0.00-0.00)\end{array}$ & $\begin{array}{l}0.02 \\
(0.16)\end{array}$ & $\begin{array}{l}57.00 \\
(37.00-77.50)\end{array}$ & $\begin{array}{l}61.80 \\
(36.44)\end{array}$ & $\begin{array}{l}0.25 \\
(0.00-1.25)\end{array}$ & $\begin{array}{l}3.73 \\
(13.16) \\
\end{array}$ & $\begin{array}{l}59.00 \\
(37.00-89.00)\end{array}$ & $\begin{array}{l}69.03 \\
(48.65) \\
\end{array}$ \\
\hline Ovary & $\begin{array}{l}0.00 \\
(0.00-0.00)\end{array}$ & $\begin{array}{l}0.00 \\
(0.00)\end{array}$ & $\begin{array}{l}16.00 \\
(5.00-41.00)\end{array}$ & $\begin{array}{l}26.68 \\
(26.94)\end{array}$ & $\begin{array}{l}0.00 \\
(0.00-0.00)\end{array}$ & $\begin{array}{l}1.76 \\
(9.89)\end{array}$ & $\begin{array}{l}16.00 \\
(5.00-45.00)\end{array}$ & $\begin{array}{l}30.49 \\
(34.00)\end{array}$ \\
\hline $\begin{array}{l}\text { Other myeloid } \\
\text { leukemia }\end{array}$ & $\begin{array}{l}0.00 \\
(0.00-0.00)\end{array}$ & $\begin{array}{l}0.11 \\
(0.58)\end{array}$ & $\begin{array}{l}17.00 \\
(3.00-67.00)\end{array}$ & $\begin{array}{l}39.96 \\
(43.74)\end{array}$ & $\begin{array}{l}0.50 \\
(0.00-6.75)\end{array}$ & $\begin{array}{l}9.65 \\
(27.23)\end{array}$ & $\begin{array}{l}34.00 \\
(5.00-81.00)\end{array}$ & $\begin{array}{l}58.63 \\
(71.01)\end{array}$ \\
\hline $\begin{array}{l}\text { Other thoracic } \\
\text { organs }\end{array}$ & $\begin{array}{l}0.00 \\
(0.00-0.00)\end{array}$ & $\begin{array}{l}0.00 \\
(0.00)\end{array}$ & $\begin{array}{l}41.50 \\
(29.00-72.00)\end{array}$ & $\begin{array}{l}58.45 \\
(50.21)\end{array}$ & $\begin{array}{l}1.75 \\
(0.50-13.50)\end{array}$ & $\begin{array}{l}8.45 \\
(12.73)\end{array}$ & $\begin{array}{l}63.00 \\
(35.50-124.00)\end{array}$ & $\begin{array}{l}80.85 \\
(59.74)\end{array}$ \\
\hline $\begin{array}{l}\text { All other cancer } \\
\text { types }\end{array}$ & $\begin{array}{l}0.00 \\
(0.00-0.00)\end{array}$ & $\begin{array}{l}0.17 \\
(1.34)\end{array}$ & $\begin{array}{l}15.50 \\
(3.00-71.50)\end{array}$ & $\begin{array}{l}43.70 \\
(52.94)\end{array}$ & $\begin{array}{l}0.00 \\
(0.00-2.00)\end{array}$ & $\begin{array}{l}5.59 \\
(16.57)\end{array}$ & $\begin{array}{l}17.50 \\
(3.50-81.50)\end{array}$ & $\begin{array}{l}61.00 \\
(82.20)\end{array}$ \\
\hline
\end{tabular}

Abbreviation: IQR, interquartile range. 
Table 4. Cost of Hospital Admission per Patient in 2018 Australian Dollars

\begin{tabular}{|c|c|c|c|c|c|c|c|c|}
\hline \multirow[b]{2}{*}{ Parameter } & \multicolumn{2}{|c|}{1 y Before Diagnosis } & \multicolumn{2}{|c|}{1 y After Diagnosis } & \multicolumn{2}{|c|}{$\begin{array}{l}2-5 \text { y After Diagnosis } \\
\text { (Annually) }\end{array}$} & \multicolumn{2}{|c|}{$\begin{array}{l}\text { Total of Years 1-5 } \\
\text { (Sum Over } 5 \text { y) }\end{array}$} \\
\hline & $\begin{array}{l}\text { Median } \\
\text { (IOR) }\end{array}$ & $\begin{array}{l}\text { Average } \\
\text { (SD) }\end{array}$ & $\begin{array}{l}\text { Median } \\
\text { (IOR) }\end{array}$ & $\begin{array}{l}\text { Average } \\
\text { (SD) }\end{array}$ & $\begin{array}{l}\text { Median } \\
\text { (IOR) }\end{array}$ & $\begin{array}{l}\text { Average } \\
\text { (SD) }\end{array}$ & $\begin{array}{l}\text { Median } \\
\text { (IQR) }\end{array}$ & $\begin{array}{l}\text { Average } \\
\text { (SD) }\end{array}$ \\
\hline \multicolumn{9}{|l|}{ Age at diagnosis } \\
\hline $0-14$ y & $\begin{array}{l}0 \\
(0-0)\end{array}$ & $\begin{array}{l}390 \\
(8,295)\end{array}$ & $\begin{array}{l}88,964 \\
(34,399-163,968)\end{array}$ & $\begin{array}{l}115,304 \\
(111,623)\end{array}$ & $\begin{array}{l}2,959 \\
(0-11,936)\end{array}$ & $\begin{array}{l}17,365 \\
(41,728)\end{array}$ & $\begin{array}{l}119,027 \\
(44,603-214,904)\end{array}$ & $\begin{array}{l}162,819 \\
(162,946)\end{array}$ \\
\hline $15-17 y$ & $\begin{array}{l}0 \\
(0-0)\end{array}$ & $\begin{array}{l}232 \\
(3,649)\end{array}$ & $\begin{array}{l}23,834 \\
(5,585-91,565)\end{array}$ & $\begin{array}{l}63,038 \\
(92,950)\end{array}$ & $\begin{array}{l}0 \\
(0-2,487)\end{array}$ & $\begin{array}{l}12,523 \\
(38,053)\end{array}$ & $\begin{array}{l}30,077 \\
(6,812-123,812)\end{array}$ & $\begin{array}{l}90,308 \\
(135,465)\end{array}$ \\
\hline \multicolumn{9}{|l|}{ By cancer type } \\
\hline $\begin{array}{l}\text { Acute lymphoblastic } \\
\text { leukemia }\end{array}$ & $\begin{array}{l}0 \\
(0-0)\end{array}$ & $\begin{array}{l}15 \\
(242)\end{array}$ & $\begin{array}{l}112,809 \\
(77,626-163,171)\end{array}$ & $\begin{array}{l}135,391 \\
(97,218)\end{array}$ & $\begin{array}{l}6,002 \\
(2,565-17,780)\end{array}$ & $\begin{array}{l}28,039 \\
(55,817)\end{array}$ & $\begin{array}{l}153,945 \\
(101,258-276,550)\end{array}$ & $\begin{array}{l}216,384 \\
(176,992)\end{array}$ \\
\hline Brain & $\begin{array}{l}0 \\
(0-0)\end{array}$ & $\begin{array}{l}483 \\
(2,719)\end{array}$ & $\begin{array}{l}80,631 \\
(29,971-159,561)\end{array}$ & $\begin{array}{l}118,412 \\
(132,587)\end{array}$ & $\begin{array}{l}4,214 \\
(0-17,767)\end{array}$ & $\begin{array}{l}16,028 \\
(31,039)\end{array}$ & $\begin{array}{l}106,378 \\
(32,775-206,305)\end{array}$ & $\begin{array}{l}150,746 \\
(161,454)\end{array}$ \\
\hline Hodgkin lymphoma & $\begin{array}{l}0 \\
(0-0)\end{array}$ & $\begin{array}{l}28 \\
(256)\end{array}$ & $\begin{array}{l}20,000 \\
(7,975-41,860)\end{array}$ & $\begin{array}{l}31,147 \\
(38,089)\end{array}$ & $\begin{array}{l}0 \\
(0-0)\end{array}$ & $\begin{array}{l}2,220 \\
(9,382)\end{array}$ & $\begin{array}{l}22,101 \\
(9,038-49,428)\end{array}$ & $\begin{array}{l}38,721 \\
(52,165)\end{array}$ \\
\hline $\begin{array}{l}\text { Non-Hodgkin } \\
\text { lymphoma }\end{array}$ & $\begin{array}{l}0 \\
(0-0)\end{array}$ & $\begin{array}{l}71 \\
(970)\end{array}$ & $\begin{array}{l}83,234 \\
(36,207-143,896)\end{array}$ & $\begin{array}{l}110,167 \\
(118,518)\end{array}$ & $\begin{array}{l}0 \\
(0-2,029)\end{array}$ & $\begin{array}{l}14,438 \\
(52,000)\end{array}$ & $\begin{array}{l}96,546 \\
(43,005-181,430)\end{array}$ & $\begin{array}{l}137,362 \\
(149,009)\end{array}$ \\
\hline Bone & $\begin{array}{l}0 \\
(0-0)\end{array}$ & $\begin{array}{l}93 \\
(841)\end{array}$ & $\begin{array}{l}134,146 \\
(56,028-196,253)\end{array}$ & $\begin{array}{l}132,032 \\
(86,638)\end{array}$ & $\begin{array}{l}2,562 \\
(0-20,436)\end{array}$ & $\begin{array}{l}18,041 \\
(33,449)\end{array}$ & $\begin{array}{l}173,238 \\
(76,781-254,313)\end{array}$ & $\begin{array}{l}177,630 \\
(131,159)\end{array}$ \\
\hline $\begin{array}{l}\text { Acute myeloid } \\
\text { leukemia }\end{array}$ & $\begin{array}{l}0 \\
(0-0)\end{array}$ & $\begin{array}{l}225 \\
(1,891)\end{array}$ & $\begin{array}{l}173,596 \\
(114,898-283,841)\end{array}$ & $\begin{array}{l}195,065 \\
(124,950)\end{array}$ & $\begin{array}{l}3,252 \\
(0-39,505)\end{array}$ & $\begin{array}{l}30,045 \\
(57,229)\end{array}$ & $\begin{array}{l}239,218 \\
(140,434-358,797)\end{array}$ & $\begin{array}{l}268,115 \\
(176,844)\end{array}$ \\
\hline $\begin{array}{l}\text { Connective tissue, } \\
\text { peripheral nerves }\end{array}$ & $\begin{array}{l}0 \\
(0-0)\end{array}$ & $\begin{array}{l}64 \\
(557)\end{array}$ & $\begin{array}{l}79,673 \\
(25,309-147,303)\end{array}$ & $\begin{array}{l}95,622 \\
(84,611)\end{array}$ & $\begin{array}{l}735 \\
(0-10,591)\end{array}$ & $\begin{array}{l}16,240 \\
(45,973)\end{array}$ & $\begin{array}{l}83,720 \\
(32,187-173,369)\end{array}$ & $\begin{array}{l}128,359 \\
(132,751)\end{array}$ \\
\hline Kidney & $\begin{array}{l}0 \\
(0-0)\end{array}$ & $\begin{array}{l}22 \\
(259)\end{array}$ & $\begin{array}{l}56,437 \\
(32,128-99,024)\end{array}$ & $\begin{array}{l}86,092 \\
(125,333)\end{array}$ & $\begin{array}{l}648 \\
(0-5,157)\end{array}$ & $\begin{array}{l}8,464 \\
(17,857)\end{array}$ & $\begin{array}{l}71,931 \\
(37,843-132,206)\end{array}$ & $\begin{array}{l}108,553 \\
(137,856)\end{array}$ \\
\hline $\begin{array}{l}\text { Other endocrine } \\
\text { glands }\end{array}$ & $\begin{array}{l}0 \\
(0-0)\end{array}$ & $\begin{array}{l}99 \\
(766)\end{array}$ & $\begin{array}{l}174,511 \\
(54,204-233,323)\end{array}$ & $\begin{array}{l}166,475 \\
(128,052)\end{array}$ & $\begin{array}{l}6,341 \\
(0-21,016)\end{array}$ & $\begin{array}{l}18,611 \\
(32,196)\end{array}$ & $\begin{array}{l}201,638 \\
(80,387-313,496)\end{array}$ & $\begin{array}{l}211,678 \\
(158,560)\end{array}$ \\
\hline Melanoma of skin & $\begin{array}{l}0 \\
(0-0)\end{array}$ & $\begin{array}{l}12 \\
(125)\end{array}$ & $\begin{array}{l}2,240 \\
(0-4,728)\end{array}$ & $\begin{array}{l}4,712 \\
(8,355)\end{array}$ & $\begin{array}{l}0 \\
(0-0)\end{array}$ & $\begin{array}{l}1,696 \\
(12,063)\end{array}$ & $\begin{array}{l}2,364 \\
(0-4,824)\end{array}$ & $\begin{array}{l}8,023 \\
(21,387)\end{array}$ \\
\hline Colon & $\begin{array}{l}0 \\
(0-0)\end{array}$ & $\begin{array}{l}5,430 \\
(45,429)\end{array}$ & $\begin{array}{l}7,418 \\
(0-14,213)\end{array}$ & $\begin{array}{l}12,956 \\
(17,864)\end{array}$ & $\begin{array}{l}0 \\
(0-0)\end{array}$ & $\begin{array}{l}880 \\
(6,351)\end{array}$ & $\begin{array}{l}7,418 \\
(0-14,213)\end{array}$ & $\begin{array}{l}14,149 \\
(21,352)\end{array}$ \\
\hline Thyroid & $\begin{array}{l}0 \\
(0-0)\end{array}$ & $\begin{array}{l}47 \\
(280)\end{array}$ & $\begin{array}{l}18,076 \\
(14,533-24,502)\end{array}$ & $\begin{array}{l}24,600 \\
(31,416)\end{array}$ & $\begin{array}{l}0 \\
(0-1,952)\end{array}$ & $\begin{array}{l}1,360 \\
(2,860)\end{array}$ & $\begin{array}{l}22,340 \\
(16,770-32,294)\end{array}$ & $\begin{array}{l}30,039 \\
(40,386)\end{array}$ \\
\hline Testis & $\begin{array}{l}0 \\
(0-0)\end{array}$ & $\begin{array}{l}33 \\
(268)\end{array}$ & $\begin{array}{l}12,818 \\
(4,446-56,604)\end{array}$ & $\begin{array}{l}41,911 \\
(69,636)\end{array}$ & $\begin{array}{l}0 \\
(0-409)\end{array}$ & $\begin{array}{l}4,488 \\
(17,296)\end{array}$ & $\begin{array}{l}16,575 \\
(4,446-71,093)\end{array}$ & $\begin{array}{l}50,829 \\
(75,916)\end{array}$ \\
\hline $\begin{array}{l}\text { Central nervous } \\
\text { system }\end{array}$ & $\begin{array}{l}0 \\
(0-0)\end{array}$ & $\begin{array}{l}1,919 \\
(6,322)\end{array}$ & $\begin{array}{l}22,388 \\
(11,237-47,513)\end{array}$ & $\begin{array}{l}46,377 \\
(78,381)\end{array}$ & $\begin{array}{l}8,759 \\
(978-18,372)\end{array}$ & $\begin{array}{l}11,910 \\
(12,508)\end{array}$ & $\begin{array}{l}57,524 \\
(24,833-123,408)\end{array}$ & $\begin{array}{l}89,703 \\
(93,518)\end{array}$ \\
\hline $\begin{array}{l}\text { III-defined and } \\
\text { unspecified site }\end{array}$ & $\begin{array}{l}0 \\
(0-0)\end{array}$ & $\begin{array}{l}328 \\
(2,544)\end{array}$ & $\begin{array}{l}110,350 \\
(33,978-176,620)\end{array}$ & $\begin{array}{l}113,038 \\
(87,461)\end{array}$ & $\begin{array}{l}1,583 \\
(0-11,545)\end{array}$ & $\begin{array}{l}13,230 \\
(23,833)\end{array}$ & $\begin{array}{l}118,998 \\
(43,115-240,476)\end{array}$ & $\begin{array}{l}155,301 \\
(134,831)\end{array}$ \\
\hline Eye & $\begin{array}{l}0 \\
(0-0)\end{array}$ & $\begin{array}{l}0 \\
(0)\end{array}$ & $\begin{array}{l}36,665 \\
(25,622-56,785)\end{array}$ & $\begin{array}{l}43,027 \\
(30,688)\end{array}$ & $\begin{array}{l}3,832 \\
(0-14,113)\end{array}$ & $\begin{array}{l}11,378 \\
(17,413)\end{array}$ & $\begin{array}{l}56,369 \\
(31,481-109,410)\end{array}$ & $\begin{array}{l}86,361 \\
(85,866)\end{array}$ \\
\hline $\begin{array}{l}\text { Other lymphatic, } \\
\text { hematopoietic }\end{array}$ & $\begin{array}{l}0 \\
(0-0)\end{array}$ & $\begin{array}{l}3,359 \\
(15,208)\end{array}$ & $\begin{array}{l}15,394 \\
(7,047-48,640)\end{array}$ & $\begin{array}{l}47,454 \\
(74,770)\end{array}$ & $\begin{array}{l}0 \\
(0-8,776)\end{array}$ & $\begin{array}{l}10,233 \\
(27,655)\end{array}$ & $\begin{array}{l}17,728 \\
(7,047-105,785)\end{array}$ & $\begin{array}{l}87,008 \\
(158,728)\end{array}$ \\
\hline Liver & $\begin{array}{l}0 \\
(0-0)\end{array}$ & $\begin{array}{l}119 \\
(764)\end{array}$ & $\begin{array}{l}93,558 \\
(59,275-143,468)\end{array}$ & $\begin{array}{l}119,033 \\
(101,722)\end{array}$ & $\begin{array}{l}994 \\
(0-4,869)\end{array}$ & $\begin{array}{l}13,050 \\
(52,128)\end{array}$ & $\begin{array}{l}102,163 \\
(61,264-162,347)\end{array}$ & $\begin{array}{l}139,880 \\
(129,826)\end{array}$ \\
\hline Ovary & $\begin{array}{l}0 \\
(0-0)\end{array}$ & $\begin{array}{l}0 \\
(0)\end{array}$ & $\begin{array}{l}28,750 \\
(10,757-62,593)\end{array}$ & $\begin{array}{l}41,131 \\
(38,757)\end{array}$ & $\begin{array}{l}0 \\
(0-0)\end{array}$ & $\begin{array}{l}3,499 \\
(20,158)\end{array}$ & $\begin{array}{l}28,750 \\
(10,757-67,248)\end{array}$ & $\begin{array}{l}48,560 \\
(57,916)\end{array}$ \\
\hline $\begin{array}{l}\text { Other myeloid } \\
\text { leukemia }\end{array}$ & $\begin{array}{l}0 \\
(0-0)\end{array}$ & $\begin{array}{l}255 \\
(1,323)\end{array}$ & $\begin{array}{l}24,339 \\
(9,221-179,247)\end{array}$ & $\begin{array}{l}86,061 \\
(101,061)\end{array}$ & $\begin{array}{l}696 \\
(0-8,593)\end{array}$ & $\begin{array}{l}22,455 \\
(59,600)\end{array}$ & $\begin{array}{l}57,534 \\
(12,441-235,245)\end{array}$ & $\begin{array}{l}131,548 \\
(145,341)\end{array}$ \\
\hline $\begin{array}{l}\text { Other thoracic } \\
\text { organs }\end{array}$ & $\begin{array}{l}0 \\
(0-0)\end{array}$ & $\begin{array}{l}0 \\
(0)\end{array}$ & $\begin{array}{l}100,557 \\
(55,242-123,343)\end{array}$ & $\begin{array}{l}119,445 \\
(95,485)\end{array}$ & $\begin{array}{l}5,853 \\
(1,296-35,077)\end{array}$ & $\begin{array}{l}16,711 \\
(22,064)\end{array}$ & $\begin{array}{l}133,897 \\
(79,643-245,969)\end{array}$ & $\begin{array}{l}167,776 \\
(122,574)\end{array}$ \\
\hline $\begin{array}{l}\text { All other cancer } \\
\text { types }\end{array}$ & $\begin{array}{l}0 \\
(0-0)\end{array}$ & $\begin{array}{l}548 \\
(5,575)\end{array}$ & $\begin{array}{l}37,732 \\
(11,585-126,099)\end{array}$ & $\begin{array}{l}90,178 \\
(124,769)\end{array}$ & $\begin{array}{l}0 \\
(0-5,237)\end{array}$ & $\begin{array}{l}10,767 \\
(29,576)\end{array}$ & $\begin{array}{l}40,415 \\
(12,577-167,510)\end{array}$ & $\begin{array}{l}124,867 \\
(171,361)\end{array}$ \\
\hline
\end{tabular}

Abbreviation: IQR, interquartile range. 
average annual per capita inpatient cost for children aged 0 to 19 years (range, $\mathrm{A} \$ 341-\mathrm{A} \$ 5,910$ ) in 2012 through $2013 .^{28}$

In NSW, LoS per hospitalization (4.4 days) among patients with childhood cancer was shorter than that reported in a 2009 US study, in which the average LoS was 12 days per hospitalization (supplemental eTable 5). ${ }^{2}$ However, the patients in NSW might have been admitted more frequently than their US counterparts, because some of the procedures performed in Australian hospitals might also be performed in outpatient settings in the United States. The US study also reported that the most common childhood cancers that required hospitalization were leukemias and brain cancers. ${ }^{2}$ In our study, we found that ALL had a higher annual number of hospital admissions (Table 2), which might be due to the patients' immunocompromised condition and complex treatment protocols that require comprehensive hospital services. ${ }^{29}$

We identified several factors that could lead to more frequent hospital admissions. Factors that might suggest more severe or more complicated cases, including cancer metastasis within 4 months of diagnosis, were associated with more frequent hospital admissions (supplemental eTable 10). Patients living in remote areas were admitted to a hospital 1.6 times more often than those living in major cities. However, there was no statistically significant difference in year $1 \mathrm{LoS}$ across all geographic distributions. Australians living in remote areas were reported to have less frequent access to nonhospital nonreferred attendances and to face the challenge of being unable to find specialists nearby. ${ }^{30}$ Our findings might reflect the compensation mechanisms for lacking access to relevant specialists in remote areas.

A study in Canada found that, in 2012, costs were much higher for patients who died within 1 year of diagnosis. ${ }^{3} \mathrm{Sim}-$ ilarly, another study in the United States reported that being diagnosed with a myeloid leukemia and death within 3 years of diagnosis were significant predictors of hospital charges. ${ }^{31}$ Consistent with other studies, ${ }^{32,33}$ we found that a diagnosis of AML incurred higher hospital costs (supplemental eTable 10). Factors that might suggest the severity of the case, including cancer metastasis within 4 months of diagnosis, were significantly associated with higher hospital costs. Early diagnosis of childhood cancers has been associated with better survival outcomes ${ }^{34,35}$ and also with reduced posttreatment comorbidities by avoiding more intense and more expensive treatment. Early diagnosis could be achieved through a combination of effective diagnosis by general practitioners $^{36}$ and the implementation of genomic sequencing into routine diagnostic procedure. ${ }^{37}$

Hospital costs were $14 \%$ higher for patients living in the most disadvantaged SEIFA IRSD area compared with those living in the least disadvantaged area (supplemental eTable 10), likely because of the higher disease burden associated with this cohort. ${ }^{38}$ Similar to other studies, ${ }^{30,38,39}$ we report that lower socioeconomic status and geographic remoteness may lead to higher hospital cost and more frequent hospital admissions. Despite the development of first National Rural Health Strategy 25 years ago, remote and rural Australians still lack access to comprehensive healthcare services. ${ }^{40}$ Recent improvement in the implementation of telehealth might prove to be a good platform with which to tackle this issue. ${ }^{41}$

Our study has some limitations. It is limited to the analysis of hospitalization and ED costs. Although our analysis accounts for the bulk of the cost of the disease, it does not include all other costs incurred beyond the hospital system, including prescription medicine, outpatient visits, allied health, access to community health services, and out-of-pocket expenses. Furthermore, our ED data were not available until 2005; therefore, ED costs incurred before 2005 were not reported (supplemental eTable 7).

\section{Conclusions}

This study highlights the high hospital service use of patients with childhood cancer and the importance of early diagnosis, with treatment of metastatic cancer being associated with higher treatment costs. Our findings also demonstrate the health inequities experienced by patients from remote and low socioeconomic status areas. Future research on spatial analysis of variations in health access by patients with cancer will provide further insights into the issue of health inequities.

\section{Acknowledgments}

We thank the Cancer Institute NSW, and the NSW Ministry of Health for access to the health data, the NSW Registry of Births Deaths and Marriages for providing access to mortality data, and the NSW Centre for Health Record Linkage for conducting record linkage. The Cause of Death Unit Record File (COD URF) is provided by the NSW Registry of Births Deaths and Marriages, NSW Coroner, and the National Coronial Information System.

Submitted March 26, 2020; final revision received December 23, 2020; accepted for publication December 23, 2020.

Published online August 6, 2021.

Author contributions: Study concept and design: All authors. Statistical analysis: Tan. Manuscript writing - original draft: Tan. Manuscript writing review and editing: All authors. Final manuscript approval: All authors.

Disclosures: The authors have disclosed that they have not received any financial consideration from any person or organization to support the preparation, analysis, results, or discussion of this article.

Correspondence: Owen Tan, MComm, GenIMPACT: Centre for Economic Impacts of Genomic Medicine, Macquarie Business School, Macquarie University, Level 3, 4ER Eastern Road, Macquarie Park, NSW 2113, Australia. Email: owen.tan@mq.edu.au 


\section{References}

1. Merrill CT, Nagamine M, Hambrick MM. Pediatric hospital stays for cancer, 2005: Statistical Brief \#37. In: Healthcare Cost and Utilization Project (HCUP) Statistical Briefs. Rockville, MD: Agency for Healthcare Research and Quality; 2006.

2. Price RA, Stranges E, Elixhauser A. Pediatric cancer hospitalizations, 2009: Statistical Brief \#132. In: Healthcare Cost and Utilization Project (HCUP) Statistical Briefs. Rockville, MD: Agency for Healthcare Research and Quality; 2012.

3. de Oliveira C, Bremner KE, Liu N, et al. Costs for childhood and adolescent cancer, 90 days prediagnosis and 1 year postdiagnosis: a population-based study in Ontario, Canada. Value Health 2017;20:345-356.

4. Australian Institute of Health and Welfare. Health System Expenditure on Cancer and Other Neoplasms in Australia 2008-09. Accessed March 13, 2020. Available at: https://www.aihw.gov.au/reports/health-welfare-expenditure/ health-system-expenditure-cancer-2008-09/contents/table-of-contents

5. Deloitte Access Economics. The economic cost of cancer in adolescents and young adults: assessing the impact. Accessed March 13, 2020. Available at: https://www2.deloitte.com/au/en/pages/economics/articles/ economics-cost-cancer-adolescents-young-adults.html

6. Lansky SB, Black JL, Cairns NU. Childhood cancer medical costs. Cancer 1983;52:762-766

7. Rahiala J, Riikonen $P$, Kekäläinen L, et al. Cost analysis of the treatment of acute childhood lymphocytic leukaemia according to Nordic protocols. Acta Paediatr 2000;89:482-487.

8. de Oliveira C, Bremner KE, Liu N, et al. Costs of cancer care in children and adolescents in Ontario, Canada. Pediatr Blood Cancer 2017;64:e26628.

9. Judicial Commission of New South Wales. Equality Before the Law Bench Book - Section 6 - children and young people. Accessed March 13, 2020. Available at: https://www.judcom.nsw.gov.au/publications/ benchbks/equality/section06.html

10. United Nations Human Rights Office of the High Commissioner. Convention on the Rights of the Child. Accessed October 7, 2020. Available at: https://www.ohchr.org/en/professionalinterest/pages/crc.aspx

11. Cancer Institute NSW. NSW Cancer Registry. Accessed March 13, 2020. Available at: https://www.cancer.nsw.gov.au/data-research/data-held-bycinsw/nsw-cancer-registry

12. Centre for Health Record Linkage. How record linkage works. Accessed March 13, 2020. Available at: https://www.cherel.org.au/how-recordlinkage-works

13. Cancer Institute NSW. Submit cancer cases to the NSW Cancer Registry. Accessed March 13, 2020. Available at: https://www.cancer.nsw.gov.au/ research-and-data/cancer-data-and-statistics/submit-cancer-cases-to-thensw-cancer-registry

14. IHPA. National hospital cost data collection, public hospitals cost report, round 20 (financial year 2015-16). Accessed March 13, 2020. Available at: https://www.ihpa.gov.au/publications/national-hospital-cost-datacollection-public-hospitals-cost-report-round-20-financial

15. Australian Government Department of Health. Archived hospital casemix protocol annual reports. HCP annual report 2015 - 2016. Accessed March 13, 2020. Available at: https://www1.health.gov.au/internet/main/ publishing.nsf/Content/health-casemix-data-collections-publicationsHCPAnnualReportsArchived

16. Australian Bureau of Statistics. Consumer Price Index, Australia. Accessed March 12, 2020. Available at: https://www.abs.gov.au/statistics/economy/ price-indexes-and-inflation/consumer-price-index-australia/latest-release

17. Australian Bureau of Statistics. The Australian Statistical Geography Standard (ASGS) Remoteness Structure. Accessed March 13, 2020. Available at: https://www.abs.gov.au/websitedbs/d3310114.nsf/home/ remoteness+structure

18. Australian Bureau of Statistics. 1033.0.55.001 - Census of Population and Housing: Socio-Economic Indexes for Areas (SEIFA), Australia, 2011. Accessed March 13, 2020. Available at: https://www.abs.gov.au/ausstats/ abs@.nsf/DetailsPage/2033.0.55.0012011?OpenDocument

19. Elsworthy AM, Claessen SM, Graham B, et al. ICD-10-AM: The International Statistical Classification of Diseases and Related Health Problems, 10th Revision, Australian modification: alphabetical index of diseases, 8 ed. Wollongong, Australia: National Casemix \& Classification Centre, Australian Health Services Research Institute; 2013.
20. Percy C, Van Holten V, Muir G, et al. New International Classification of Diseases for Oncology (ICD-O, 2nd Edition) and the neoplasm chapter of the 10th Revision of the International Classification of Diseases (ICD-10) [abstract]. Lab Invest 1990;62:A113.

21. Goldsbury D, Weber $M$, Yap $S$, et al. Identifying incident colorectal and lung cancer cases in health service utilisation databases in Australia: a validation study. BMC Med Inform Decis Mak 2017;17:23.

22. Luo $\mathrm{Q}, \mathrm{Yu} X \mathrm{X}$, Smith DP, et al. Cancer-related hospitalisations and 'unknown' stage prostate cancer: a population-based record linkage study. BMJ Open 2017;7:e014259.

23. Australian Institute of Health and Welfare. Australian refined diagnosisrelated groups (AR-DRG) data cubes. Accessed March 13, 2020. Available at: aihw.gov.au/reports/hospitals/ar-drg-data-cubes/contents/data-cubes

24. Baade PD, Youlden DR, Valery PC, et al. Population-based survival estimates for childhood cancer in Australia during the period 1997-2006. Br J Cancer 2010;103:1663-1670.

25. National Cancer Control Indicators. Relative survival by stage at diagnosis 2011-2016, a snapshot in time. Accessed March 13, 2020. Available at: https://ncci.canceraustralia.gov.au/features/relative-survival-stagediagnosis-2011\%E2\%80\%932016-snapshot-time

26. Working Group. International rules for multiple primary cancers (ICD-0 third edition): working group report. Eur J Cancer Prev 2005;14:307-308.

27. Mitchell RJ, Curtis K, Foster K. A 10-year review of child injury hospitalisations, health outcomes and treatment costs in Australia. Inj Prev 2018;24: 344-350.

28. Australian Institute of Health and Welfare. Australian health expendituredemographics and diseases. Hospital admitted patient expenditure 2004-05 to 2012-13. Accessed March 13, 2020. Available at: https://www. aihw.gov.au/getmedia/fcccbe83-7953-424d-a8e5-c3859723b5c3/aihwhwe-069.pdf.aspx?inline=true

29. Wilkes JJ, Hennessy S, Xiao R, et al. Volume-outcome relationships in pediatric acute lymphoblastic leukemia: association between hospital pediatric and pediatric oncology volume with mortality and intensive care resources during initial therapy. Clin Lymphoma Myeloma Leuk 2016;16:404-410.e1.

30. Australian Institute of Health and Welfare. Rural \& remote health. Accessed March 13, 2020. Available at: https://www.aihw.gov.au/reports/ruralremote-australians/rural-remote-health/contents/summary

31. Rosenman MB, Vik T, Hui SL, et al. Hospital resource utilization in childhood cancer. J Pediatr Hematol Oncol 2005;27:295-300.

32. Mahmoud D, Skikne BS, Kucmin-Bemelmans I, et al. Overall economic burden of total treatment costs in acute myeloid leukemia throughout the course of the disease [abstract]. Blood 2012;120:3614.

33. Sprehe MR, Johnson R. Using chargemaster data to understand childhood leukemia costs [abstract]. Blood 2016;128:5938.

34. Gupta S, Howard SC, Hunger SP, et al. Treating childhood cancer in lowand middle-income countries. In: Gelband H, Jha P, Sankaranarayanan R, et al, eds. Cancer: Disease Control Priorities, Vol. 3. 3rd ed. Washington, DC: International Bank for Reconstruction and Development/World Bank; 2015:121-146.

35. GBD 2017 Childhood Cancer Collaborators. The global burden of child hood and adolescent cancer in 2017: an analysis of the Global Burden of Disease Study 2017. Lancet Oncol 2019;20:1211-1225.

36. Ahrensberg JM, Olesen F, Hansen RP, et al. Childhood cancer and factors related to prolonged diagnostic intervals: a Danish population-based study. Br J Cancer 2013;108:1280-1287.

37. Wise J. Genome sequencing of children promises a new era in oncology. BMJ 2019;364:I105.

38. Tervonen HE, Aranda S, Roder D, et al. Cancer survival disparities worsening by socio-economic disadvantage over the last 3 decades in new South Wales, Australia. BMC Public Health 2017;17:691.

39. Marmot M. Social determinants of health inequalities. Lancet 2005;365: 1099-1104.

40. Wakerman J, Humphreys JS. "Better health in the bush": why we urgently need a national rural and remote health strategy. Med J Aust 2019:210: 202-203.e1.

41. Fisk M, Livingstone A, Pit SW. Telehealth in the context of COVID-19: changing perspectives in Australia, the United Kingdom, and the United States. J Med Internet Res 2020;22:e19264. 
Supplemental online content for:

\section{An Analysis of Hospital Costs for Childhood Cancer Care}

Owen Tan, MComm; Deborah J. Schofield, PhD; and Rupendra Shrestha, PhD

J Natl Compr Canc Netw 2022;20(2):126-135

eTable 1: Details of Datasets and Percent of Patients in the NSW CCR With Matching Records in Other Linked Datasets

eTable 2: Reporting Categories of Cancer Groups

eTable 3: Cancer Types Included in "All Other Cancer Types"

eTable 4: Annual Frequency of Emergency Department Presentations per Patient

eTable 5: Average Length of Stay in Hospital, by Age at Diagnosis

eTable 6: Number of Years Spent in Hospital System

eTable 7: Cost of Emergency Department Presentation per Patient in 2018 Australian Dollars

eTable 8: Cost of Cancer-Related Hospital Admissions and Emergency Department Presentations in Australian Dollars

eTable 9: Principal Diagnosis of Hospital Admissions Within 5 Years of Being Diagnosed With Cancer

eTable 10: Variables Associated With Number of Hospital Admissions, Hospital LOS, and Cost of Hospital Admissions Within First Year of Cancer Diagnosis 


\begin{tabular}{|c|c|c|c|}
\hline Datasets & $\begin{array}{l}\text { No. of } \\
\text { Individuals }\end{array}$ & $\begin{array}{l}\% \text { of NSW Central } \\
\text { Cancer Registry }\end{array}$ & Data Available \\
\hline NSW Central Cancer Registry & 2,966 & $100 \%$ & July 1, 2001-December 31, 2012 \\
\hline NSW Admitted Patient Data Collection & 2,844 & $96 \%$ & July 1, 2001-March 31, 2017 \\
\hline NSW Emergency Department Data Collection & 1,929 & $65 \%$ & January 1, 2005-March 31, 2017 \\
\hline NSW Registry of Births, Deaths \& Marriages & 541 & $18 \%$ & July 1, 2001-March 31, 2017 \\
\hline NSW Cause of Death Unit Record File & 527 & $18 \%$ & July 1, 2001-December 31, 2015 \\
\hline
\end{tabular}

Note: $96 \%$ of patients in the NSW Central Cancer Registry have a matching hospital admission record, and $65 \%$ of patients in the registry have a matching emergency department record.

Abbreviation: NSW, New South Wales.

\section{eTable 2. Reporting Categories of Cancer Groups}

\begin{tabular}{|c|c|c|}
\hline Topology Groups & Cancer Types & ICD-O-3 Codes \\
\hline \multirow[t]{3}{*}{ Skin } & C00 Lip & $\mathrm{COO}$ \\
\hline & C43 Melanoma of skin & C44 and M872x-M879x \\
\hline & C46 Kaposi's sarcoma & M914x \\
\hline \multirow[t]{9}{*}{ Head and neck } & C012 Tongue & $\mathrm{C} 01, \mathrm{C} 02$ \\
\hline & C036 Mouth & C03-C06 \\
\hline & C078 Salivary glands & $\mathrm{C} 07, \mathrm{C} 08$ \\
\hline & C0910 Oropharynx & $\mathrm{C} 09, \mathrm{C} 10$ \\
\hline & C11 Nasopharynx & C11 \\
\hline & C123 Hypopharynx & $\mathrm{C} 12, \mathrm{C} 13$ \\
\hline & C14 Other oral cavity and pharynx & C14 \\
\hline & C301 Nose, sinuses, etc. & C30, C31 \\
\hline & C32 Larynx & C32 \\
\hline Eye & C69 Eye & C69 \\
\hline \multirow[t]{6}{*}{ Upper gastrointestinal } & C15 Esophagus & C15 \\
\hline & C16 Stomach & C16 \\
\hline & C17 Small intestine & C17 \\
\hline & C22 Liver & $\mathrm{C} 22$ \\
\hline & C234 Gallbladder & $\mathrm{C} 23, \mathrm{C} 24$ \\
\hline & C25 Pancreas & $\mathrm{C} 25$ \\
\hline \multirow[t]{2}{*}{ Colorectal } & C18 Colon & C18 \\
\hline & C1921 Rectum, rectosigmoid, anus & C19-C21 \\
\hline \multirow[t]{3}{*}{ Respiratory } & C334 Lung & C33, C34 \\
\hline & C378 Other thoracic organs & C37, C38 \\
\hline & C45 Mesothelioma & M905x \\
\hline \multirow[t]{2}{*}{ Bone and connective tissue } & C401 Bone & C40, C41 \\
\hline & C479 Connective tissue, peripheral nerves & C47, C49 \\
\hline Breast & C50 Breast & C50 \\
\hline \multirow[t]{5}{*}{ Gynecologic } & C53 Cervix & $\mathrm{C} 53$ \\
\hline & C545 Uterus, body and NOS & C54, C55 \\
\hline & C567 Ovary & C56, C57.0-7 \\
\hline & C58 Placenta & C58 \\
\hline & C59 Other female genital organs & C51, C52, C57.8-9 \\
\hline
\end{tabular}




\begin{tabular}{|c|c|c|}
\hline Topology Groups & Cancer Types & ICD-0-3 Codes \\
\hline \multirow[t]{5}{*}{ Urogenital } & $\$ 1$ Prostate & $\mathrm{C} 61$ \\
\hline & \$2 Testis & C62 \\
\hline & $\$ 360$ Other male genital organs & $\mathrm{C} 60, \mathrm{C} 63$ \\
\hline & \$48 Kidney & $\mathrm{C} 64-\mathrm{C} 66, \mathrm{C} 68$ \\
\hline & C67 Bladder & $\mathrm{C} 67$ \\
\hline \multirow[t]{2}{*}{ Neurologic } & C71 Brain & C71 \\
\hline & C72 Central nervous system & $\mathrm{C} 70, \mathrm{C} 72$ \\
\hline \multirow[t]{2}{*}{ Thyroid/Endocrine } & C73 Thyroid & $\mathrm{C} 73$ \\
\hline & C745 Other endocrine glands & $\mathrm{C} 74, \mathrm{C} 75$ \\
\hline \multirow[t]{11}{*}{ Lymphohematopoietic } & C81 Hodgkin lymphoma & M965x-M966x \\
\hline & 32 Non-Hodgkin lymphoma & M959x, M967x-M972x, M974x \\
\hline & 3890 Multiple myeloma & M973x, M976x \\
\hline & 710 Acute lymphoblastic leukemia & M9821 \\
\hline & 719 Other lymphoid leukemia's & M9820, M9822-M9827, M994 \\
\hline & 720 Acute myeloid leukemia & M9861 \\
\hline & 729 Other myeloid leukemia & $\begin{array}{l}\text { M9860, M9862-M9868, M987x-M988x, } \\
\text { M9930, M9987 }\end{array}$ \\
\hline & 74 Other specified leukemia's & M984x, M985x, M989x-M993x \\
\hline & 75 Unspecified leukemia's & M980x \\
\hline & 95 Other lymphatic hematopoietic & $\begin{array}{l}\text { C42, C77, and M974x, M975x, } \\
\text { M995x-M997x [excluding M9963] }\end{array}$ \\
\hline & M96 Myelodysplasia & M998 \\
\hline Cancer unknown primary & C98 Undefined and unspecified site & C26, C39, C48, C76, C80 \\
\hline
\end{tabular}

Note: Tabulation categories for cancer site were derived from ICD-O-3 topography site and morphology. The categorization was originally developed to allow consistent reporting of cancer registry data. " $x$ " in the ICD-O-3 codes represents the numbers 0 through 9.

Abbreviation: NOS, not otherwise specified.

\section{eTable 3. Cancer Types Included in "All Other Cancer Types"}

\begin{tabular}{|llll}
\hline Lip & Tongue & Mouth & Salivary gland \\
\hline Nasopharynx & Other oral cavity and pharynx & Stomach & Small intestine \\
\hline Rectum & Pancreas & Nose/Sinuses & Larynx \\
\hline Lung & Breast & Cervix & Uterus \\
\hline Other female genital organs & Prostate & Other male genital organs & Bladder \\
\hline Multiple myeloma & Other lymphoid leukemias & Other specified leukemias & Unspecified leukemias \\
\hline Myelodysplasia & & & \\
\hline
\end{tabular}




\begin{tabular}{|c|c|c|c|c|c|c|c|c|}
\hline \multirow[b]{2}{*}{ Parameter } & \multicolumn{2}{|c|}{1 y Before Diagnosis } & \multicolumn{2}{|c|}{1 y After Diagnosis } & \multicolumn{2}{|c|}{$\begin{array}{c}\text { 2-5 y After Diagnosis } \\
\text { (Annually) }\end{array}$} & \multicolumn{2}{|c|}{$\begin{array}{c}\text { Total of Years 1-5 } \\
\text { (Sum Over } 5 \text { y) }\end{array}$} \\
\hline & $\begin{array}{l}\text { Median } \\
\text { (IQR) }\end{array}$ & $\begin{array}{l}\text { Average } \\
\text { (SD) }\end{array}$ & $\begin{array}{l}\text { Median } \\
\text { (IQR) }\end{array}$ & $\begin{array}{l}\text { Average } \\
\text { (SD) }\end{array}$ & $\begin{array}{l}\text { Median } \\
\text { (IQR) }\end{array}$ & $\begin{array}{l}\text { Average } \\
\text { (SD) }\end{array}$ & $\begin{array}{l}\text { Median } \\
\text { (IQR) }\end{array}$ & $\begin{array}{l}\text { Average } \\
\text { (SD) }\end{array}$ \\
\hline \multicolumn{9}{|l|}{ Age at diagnosis } \\
\hline $0-14 y$ & $\begin{array}{l}0.00 \\
(0.00-0.00)\end{array}$ & $\begin{array}{l}0.39 \\
(0.90)\end{array}$ & $\begin{array}{l}2.00 \\
(0.00-6.00)\end{array}$ & $\begin{array}{l}3.41 \\
(4.02)\end{array}$ & $\begin{array}{l}0.50 \\
(0.00-1.25)\end{array}$ & $\begin{array}{l}0.89 \\
(1.23)\end{array}$ & $\begin{array}{l}4.00 \\
(1.00-9.00)\end{array}$ & $\begin{array}{l}6.39 \\
(6.79)\end{array}$ \\
\hline $15-17$ y & $\begin{array}{l}0.00 \\
(0.00-0.00)\end{array}$ & $\begin{array}{l}0.31 \\
(0.79)\end{array}$ & $\begin{array}{l}0.00 \\
(0.00-2.00)\end{array}$ & $\begin{array}{l}1.55 \\
(2.50)\end{array}$ & $\begin{array}{l}0.25 \\
(0.00-0.75)\end{array}$ & $\begin{array}{l}0.60 \\
(1.03)\end{array}$ & $\begin{array}{l}2.00 \\
(0.00-5.00)\end{array}$ & $\begin{array}{l}3.45 \\
(4.92)\end{array}$ \\
\hline \multicolumn{9}{|l|}{ By cancer type } \\
\hline $\begin{array}{l}\text { Acute lymphoblastic } \\
\text { leukemia }\end{array}$ & $\begin{array}{l}0.00 \\
(0.00-0.00)\end{array}$ & $\begin{array}{l}0.35 \\
(0.80)\end{array}$ & $\begin{array}{l}3.00 \\
(0.00-7.00)\end{array}$ & $\begin{array}{l}4.29 \\
(4.38)\end{array}$ & $\begin{array}{l}0.75 \\
(0.25-1.50)\end{array}$ & $\begin{array}{l}1.18 \\
(1.39)\end{array}$ & $\begin{array}{l}7.00 \\
(2.00-12.00)\end{array}$ & $\begin{array}{l}8.46 \\
(7.82)\end{array}$ \\
\hline Brain & $\begin{array}{l}0.00 \\
(0.00-0.00)\end{array}$ & $\begin{array}{l}0.39 \\
(0.86)\end{array}$ & $\begin{array}{l}2.00 \\
(0.00-4.00)\end{array}$ & $\begin{array}{l}2.88 \\
(3.49)\end{array}$ & $\begin{array}{l}0.25 \\
(0.00-1.00)\end{array}$ & $\begin{array}{l}0.87 \\
(1.27)\end{array}$ & $\begin{array}{l}3.00 \\
(1.00-7.00)\end{array}$ & $\begin{array}{l}5.04 \\
(5.80)\end{array}$ \\
\hline Hodgkin lymphoma & $\begin{array}{l}0.00 \\
(0.00-0.00)\end{array}$ & $\begin{array}{l}0.33 \\
(0.81)\end{array}$ & $\begin{array}{l}1.00 \\
(0.00-3.00)\end{array}$ & $\begin{array}{l}1.90 \\
(2.63)\end{array}$ & $\begin{array}{l}0.25 \\
(0.00-0.50)\end{array}$ & $\begin{array}{l}0.37 \\
(0.64)\end{array}$ & $\begin{array}{l}2.00 \\
(0.00-5.00)\end{array}$ & $\begin{array}{l}3.37 \\
(4.35)\end{array}$ \\
\hline $\begin{array}{l}\text { Non-Hodgkin } \\
\text { lymphoma }\end{array}$ & $\begin{array}{l}0.00 \\
(0.00-0.00)\end{array}$ & $\begin{array}{l}0.46 \\
(1.06)\end{array}$ & $\begin{array}{l}2.00 \\
(0.00-5.00)\end{array}$ & $\begin{array}{l}3.04 \\
(3.60)\end{array}$ & $\begin{array}{l}0.25 \\
(0.00-0.75)\end{array}$ & $\begin{array}{l}0.66 \\
(1.00)\end{array}$ & $\begin{array}{l}4.00 \\
(1.00-8.00)\end{array}$ & $\begin{array}{l}5.27 \\
(5.61)\end{array}$ \\
\hline Bone & $\begin{array}{l}0.00 \\
(0.00-0.00)\end{array}$ & $\begin{array}{l}0.22 \\
(0.56)\end{array}$ & $\begin{array}{l}1.00 \\
(0.00-4.00)\end{array}$ & $\begin{array}{l}2.24 \\
(3.26)\end{array}$ & $\begin{array}{l}0.25 \\
(0.00-0.75)\end{array}$ & $\begin{array}{l}0.61 \\
(0.98)\end{array}$ & $\begin{array}{l}2.00 \\
(1.00-5.00)\end{array}$ & $\begin{array}{l}4.05 \\
(5.14)\end{array}$ \\
\hline $\begin{array}{l}\text { Acute myeloid } \\
\text { leukemia }\end{array}$ & $\begin{array}{l}0.00 \\
(0.00-0.00)\end{array}$ & $\begin{array}{l}0.36 \\
(0.86)\end{array}$ & $\begin{array}{l}3.00 \\
(0.00-6.00)\end{array}$ & $\begin{array}{l}3.71 \\
(3.78)\end{array}$ & $\begin{array}{l}0.50 \\
(0.00-1.50)\end{array}$ & $\begin{array}{l}1.03 \\
(1.25)\end{array}$ & $\begin{array}{l}5.00 \\
(2.00-10.00)\end{array}$ & $\begin{array}{l}7.08 \\
(7.33)\end{array}$ \\
\hline $\begin{array}{l}\text { Connective tissue, } \\
\text { peripheral nerves }\end{array}$ & $\begin{array}{l}0.00 \\
(0.00-0.00)\end{array}$ & $\begin{array}{l}0.40 \\
(1.10)\end{array}$ & $\begin{array}{l}1.00 \\
(0.00-4.00)\end{array}$ & $\begin{array}{l}2.89 \\
(4.05)\end{array}$ & $\begin{array}{l}0.25 \\
(0.00-1.00)\end{array}$ & $\begin{array}{l}0.92 \\
(1.57)\end{array}$ & $\begin{array}{l}3.00 \\
(1.00-9.00)\end{array}$ & $\begin{array}{l}5.41 \\
(6.35)\end{array}$ \\
\hline Kidney & $\begin{array}{l}0.00 \\
(0.00-0.00)\end{array}$ & $\begin{array}{l}0.33 \\
(0.79)\end{array}$ & $\begin{array}{l}3.00 \\
(0.00-6.00)\end{array}$ & $\begin{array}{l}3.52 \\
(3.73)\end{array}$ & $\begin{array}{l}0.25 \\
(0.00-1.00)\end{array}$ & $\begin{array}{l}0.63 \\
(0.90)\end{array}$ & $\begin{array}{l}5.00 \\
(1.00-8.00)\end{array}$ & $\begin{array}{l}5.66 \\
(5.32)\end{array}$ \\
\hline $\begin{array}{l}\text { Other endocrine } \\
\text { glands }\end{array}$ & $\begin{array}{l}0.00 \\
(0.00-1.00)\end{array}$ & $\begin{array}{l}0.65 \\
(1.09)\end{array}$ & $\begin{array}{l}4.00 \\
(0.00-7.00)\end{array}$ & $\begin{array}{l}4.68 \\
(4.96)\end{array}$ & $\begin{array}{l}0.75 \\
(0.13-1.75)\end{array}$ & $\begin{array}{l}1.20 \\
(1.54)\end{array}$ & $\begin{array}{l}7.00 \\
(2.00-11.00)\end{array}$ & $\begin{array}{l}7.79 \\
(6.84)\end{array}$ \\
\hline Melanoma of skin & $\begin{array}{l}0.00 \\
(0.00-0.00)\end{array}$ & $\begin{array}{l}0.19 \\
(0.63)\end{array}$ & $\begin{array}{l}0.00 \\
(0.00-0.00)\end{array}$ & $\begin{array}{l}0.37 \\
(1.03)\end{array}$ & $\begin{array}{l}0.25 \\
(0.00-0.50)\end{array}$ & $\begin{array}{l}0.52 \\
(1.00)\end{array}$ & $\begin{array}{l}1.00 \\
(0.00-2.00)\end{array}$ & $\begin{array}{l}2.35 \\
(4.23)\end{array}$ \\
\hline Colon & $\begin{array}{l}0.00 \\
(0.00-1.00)\end{array}$ & $\begin{array}{l}0.70 \\
(1.38)\end{array}$ & $\begin{array}{l}1.00 \\
(0.00-3.00)\end{array}$ & $\begin{array}{l}1.84 \\
(1.95)\end{array}$ & $\begin{array}{l}0.25 \\
(0.00-1.00)\end{array}$ & $\begin{array}{l}0.59 \\
(0.90)\end{array}$ & $\begin{array}{l}3.00 \\
(1.00-6.00)\end{array}$ & $\begin{array}{l}4.14 \\
(4.85)\end{array}$ \\
\hline Thyroid & $\begin{array}{l}0.00 \\
(0.00-0.00)\end{array}$ & $\begin{array}{l}0.24 \\
(0.58)\end{array}$ & $\begin{array}{l}0.00 \\
(0.00-0.50)\end{array}$ & $\begin{array}{l}0.54 \\
(1.63)\end{array}$ & $\begin{array}{l}0.00 \\
(0.00-0.38)\end{array}$ & $\begin{array}{l}0.31 \\
(0.64)\end{array}$ & $\begin{array}{l}1.00 \\
(0.00-2.00)\end{array}$ & $\begin{array}{l}1.78 \\
(3.85)\end{array}$ \\
\hline Testis & $\begin{array}{l}0.00 \\
(0.00-0.00)\end{array}$ & $\begin{array}{l}0.18 \\
(0.58)\end{array}$ & $\begin{array}{l}0.00 \\
(0.00-2.00)\end{array}$ & $\begin{array}{l}1.48 \\
(2.92)\end{array}$ & $\begin{array}{l}0.25 \\
(0.00-0.50)\end{array}$ & $\begin{array}{l}0.48 \\
(0.79)\end{array}$ & $\begin{array}{l}2.00 \\
(0.00-4.00)\end{array}$ & $\begin{array}{l}3.01 \\
(3.86)\end{array}$ \\
\hline $\begin{array}{l}\text { Central nervous } \\
\text { system }\end{array}$ & $\begin{array}{l}0.00 \\
(0.00-0.00)\end{array}$ & $\begin{array}{l}0.39 \\
(0.94)\end{array}$ & $\begin{array}{l}0.00 \\
(0.00-2.00)\end{array}$ & $\begin{array}{l}1.65 \\
(2.46)\end{array}$ & $\begin{array}{l}0.50 \\
(0.25-1.00)\end{array}$ & $\begin{array}{l}0.71 \\
(0.84)\end{array}$ & $\begin{array}{l}2.00 \\
(1.00-6.00)\end{array}$ & $\begin{array}{l}4.26 \\
(4.62)\end{array}$ \\
\hline $\begin{array}{l}\text { III-defined and } \\
\text { unspecified site }\end{array}$ & $\begin{array}{l}0.00 \\
(0.00-0.00)\end{array}$ & $\begin{array}{l}0.28 \\
(0.56)\end{array}$ & $\begin{array}{l}1.00 \\
(0.00-5.00)\end{array}$ & $\begin{array}{l}2.95 \\
(4.00)\end{array}$ & $\begin{array}{l}0.50 \\
(0.25-1.25)\end{array}$ & $\begin{array}{l}0.81 \\
(1.00)\end{array}$ & $\begin{array}{l}3.00 \\
(1.00-10.00)\end{array}$ & $\begin{array}{l}5.84 \\
(6.36) \\
\end{array}$ \\
\hline Eye & $\begin{array}{l}0.00 \\
(0.00-0.00)\end{array}$ & $\begin{array}{l}0.32 \\
(0.82)\end{array}$ & $\begin{array}{l}1.00 \\
(0.00-3.00)\end{array}$ & $\begin{array}{l}1.78 \\
(2.25)\end{array}$ & $\begin{array}{l}0.25 \\
(0.00-0.75)\end{array}$ & $\begin{array}{l}0.54 \\
(0.74)\end{array}$ & $\begin{array}{l}2.00 \\
(1.00-5.00)\end{array}$ & $\begin{array}{l}3.85 \\
(4.15)\end{array}$ \\
\hline $\begin{array}{l}\text { Other lymphatic, } \\
\text { hematopoietic }\end{array}$ & $\begin{array}{l}0.00 \\
(0.00-0.00)\end{array}$ & $\begin{array}{l}0.59 \\
(1.38)\end{array}$ & $\begin{array}{l}1.00 \\
(0.00-3.00)\end{array}$ & $\begin{array}{l}1.94 \\
(3.04)\end{array}$ & $\begin{array}{l}0.50 \\
(0.25-1.00)\end{array}$ & $\begin{array}{l}0.71 \\
(0.79)\end{array}$ & $\begin{array}{l}3.00 \\
(2.00-6.00)\end{array}$ & $\begin{array}{l}4.67 \\
(4.46)\end{array}$ \\
\hline Liver & $\begin{array}{l}0.00 \\
(0.00-1.00)\end{array}$ & $\begin{array}{l}0.46 \\
(0.84)\end{array}$ & $\begin{array}{l}3.00 \\
(0.00-7.00)\end{array}$ & $\begin{array}{l}4.14 \\
(4.10)\end{array}$ & $\begin{array}{l}0.75 \\
(0.00-1.13)\end{array}$ & $\begin{array}{l}1.00 \\
(1.45)\end{array}$ & $\begin{array}{l}5.00 \\
(2.00-13.00)\end{array}$ & $\begin{array}{l}7.59 \\
(8.06)\end{array}$ \\
\hline Ovary & $\begin{array}{l}0.00 \\
(0.00-0.00)\end{array}$ & $\begin{array}{l}0.15 \\
(0.36)\end{array}$ & $\begin{array}{l}1.00 \\
(0.00-2.00)\end{array}$ & $\begin{array}{l}1.85 \\
(3.93)\end{array}$ & $\begin{array}{l}0.00 \\
(0.00-0.50)\end{array}$ & $\begin{array}{l}0.46 \\
(1.13)\end{array}$ & $\begin{array}{l}2.00 \\
(0.00-3.50)\end{array}$ & $\begin{array}{l}3.68 \\
(8.13)\end{array}$ \\
\hline $\begin{array}{l}\text { Other myeloid } \\
\text { leukemia }\end{array}$ & $\begin{array}{l}0.00 \\
(0.00-0.00)\end{array}$ & $\begin{array}{l}0.19 \\
(0.48)\end{array}$ & $\begin{array}{l}2.00 \\
(0.00-3.00)\end{array}$ & $\begin{array}{l}1.68 \\
(1.49)\end{array}$ & $\begin{array}{l}0.25 \\
(0.00-1.25)\end{array}$ & $\begin{array}{l}0.82 \\
(1.24)\end{array}$ & $\begin{array}{l}3.00 \\
(1.00-6.00)\end{array}$ & $\begin{array}{l}4.56 \\
(5.09)\end{array}$ \\
\hline $\begin{array}{l}\text { Other thoracic } \\
\text { organs }\end{array}$ & $\begin{array}{l}0.00 \\
(0.00-1.00)\end{array}$ & $\begin{array}{l}0.75 \\
(1.25)\end{array}$ & $\begin{array}{l}4.00 \\
(0.00-7.00)\end{array}$ & $\begin{array}{l}4.22 \\
(4.17)\end{array}$ & $\begin{array}{l}0.38 \\
(0.25-0.75)\end{array}$ & $\begin{array}{l}0.74 \\
(0.93)\end{array}$ & $\begin{array}{l}6.00 \\
(2.00-9.00)\end{array}$ & $\begin{array}{l}6.67 \\
(5.76)\end{array}$ \\
\hline $\begin{array}{l}\text { All other cancer } \\
\text { types }\end{array}$ & $\begin{array}{l}0.00 \\
(0.00-1.00)\end{array}$ & $\begin{array}{l}0.51 \\
(1.02)\end{array}$ & $\begin{array}{l}0.00 \\
(0.00-3.00)\end{array}$ & $\begin{array}{l}2.15 \\
(3.24)\end{array}$ & $\begin{array}{l}0.25 \\
(0.00-0.75)\end{array}$ & $\begin{array}{l}0.63 \\
(0.98)\end{array}$ & $\begin{array}{l}2.00 \\
(0.00-6.00)\end{array}$ & $\begin{array}{l}4.32 \\
(5.45)\end{array}$ \\
\hline
\end{tabular}

Abbreviation: IQR, interquartile range. 


\begin{tabular}{|ll|}
\hline $\begin{array}{l}\text { eTable 5. Average Length of Stay in Hospital, } \\
\text { by Age at Diagnosis }\end{array}$ \\
\hline Age at Diagnosis & Average (SD), d \\
\hline All age groups & $4.40(9.86)$ \\
\hline $0-4$ y & $4.05(9.67)$ \\
\hline $5-9$ y & $4.14(9.27)$ \\
\hline $10-14$ y & $4.86(9.49)$ \\
\hline $15-17 y$ & $5.32(11.62)$ \\
\hline
\end{tabular}

\begin{tabular}{|c|c|c|}
\hline & Median (IQR) & Average (SD) \\
\hline \multicolumn{3}{|l|}{ Age at diagnosis } \\
\hline $0-14 y$ & $1.84(0.63-2.49)$ & $1.78(1.37)$ \\
\hline $15-17 y$ & $0.48(0.00-1.37)$ & $0.97(1.25)$ \\
\hline \multicolumn{3}{|l|}{ By cancer type } \\
\hline Acute lymphoblastic leukemia & $2.05(1.99-2.63)$ & $2.33(1.05)$ \\
\hline Brain & $1.02(0.24-2.64)$ & $1.53(1.49)$ \\
\hline Hodgkin lymphoma & $0.46(0.12-0.77)$ & $0.69(0.9)$ \\
\hline Non-Hodgkin lymphoma & $0.67(0.32-1.69)$ & $1.12(1.12)$ \\
\hline Bone & $1.17(0.75-2.72)$ & $1.71(1.32)$ \\
\hline Acute myeloid leukemia & $1.17(0.52-2.29)$ & $1.64(1.42)$ \\
\hline Connective tissue, peripheral nerves & $1.14(0.53-2.2)$ & $1.48(1.33)$ \\
\hline Kidney & $0.98(0.61-1.67)$ & $1.28(1.08)$ \\
\hline Other endocrine glands & $1.58(0.81-3.08)$ & $1.88(1.31)$ \\
\hline Melanoma of skin & $0(0-0.15)$ & $0.46(1.12)$ \\
\hline Colon & $0(0-0.09)$ & $0.19(0.64)$ \\
\hline Thyroid & $0.27(0.12-1.61)$ & $0.99(1.28)$ \\
\hline Testis & $0.38(0-1.05)$ & $0.7(0.87)$ \\
\hline Central nervous system & $3.35(0.59-4.37)$ & $2.72(1.86)$ \\
\hline III-defined and unspecified site & $1.22(0.17-2.86)$ & $1.66(1.52)$ \\
\hline Eye & $2.36(1.18-3.78)$ & $2.48(1.53)$ \\
\hline Other lymphatic, hematopoietic & $0.63(0-2.39)$ & $1.47(1.72)$ \\
\hline Liver & $0.86(0.45-1.66)$ & $1.24(1.16)$ \\
\hline Ovary & $0.17(0-0.68)$ & $0.41(0.52)$ \\
\hline Other myeloid leukemia & $0.85(0-1.98)$ & $1.39(1.58)$ \\
\hline Other thoracic organs & $1.54(0.9-3.12)$ & $1.89(1.43)$ \\
\hline All other cancer types & $0.86(0.06-2.01)$ & $1.37(1.48)$ \\
\hline
\end{tabular}

Abbreviation: IQR, interquartile range.

${ }^{a}$ Between first cancer-related admission and last cancer-related separation. 


\begin{tabular}{|c|c|c|c|c|c|c|c|c|}
\hline \multirow[b]{2}{*}{ Parameter } & \multicolumn{2}{|c|}{1 y Before Diagnosis } & \multicolumn{2}{|c|}{1 y After Diagnosis } & \multicolumn{2}{|c|}{$\begin{array}{c}2-5 \text { y After Diagnosis } \\
\text { (Annually) }\end{array}$} & \multicolumn{2}{|c|}{$\begin{array}{l}\text { Total of Years 1-5 } \\
\text { (Sum Over } 5 \text { y) }\end{array}$} \\
\hline & $\begin{array}{l}\text { Median } \\
\text { (IQR) }\end{array}$ & $\begin{array}{l}\text { Average } \\
\text { (SD) }\end{array}$ & $\begin{array}{l}\text { Median } \\
\text { (IQR) }\end{array}$ & $\begin{array}{l}\text { Average } \\
\text { (SD) }\end{array}$ & $\begin{array}{l}\text { Median } \\
\text { (IQR) }\end{array}$ & $\begin{array}{l}\text { Average } \\
\text { (SD) }\end{array}$ & $\begin{array}{l}\text { Median } \\
\text { (IQR) }\end{array}$ & $\begin{array}{l}\text { Average } \\
\text { (SD) }\end{array}$ \\
\hline \multicolumn{9}{|l|}{ Age at diagnosis } \\
\hline $0-14$ y & $\begin{array}{l}0 \\
(0-0)\end{array}$ & $\begin{array}{l}227 \\
(580)\end{array}$ & $\begin{array}{l}1,782 \\
(0-4,902)\end{array}$ & $\begin{array}{l}2,936 \\
(3,407)\end{array}$ & $\begin{array}{l}251 \\
(0-804)\end{array}$ & $\begin{array}{l}637 \\
(1,006)\end{array}$ & $\begin{array}{l}3,252 \\
(787-7,469)\end{array}$ & $\begin{array}{l}5,033 \\
(5,520)\end{array}$ \\
\hline $15-17$ y & $\begin{array}{l}0 \\
(0-0)\end{array}$ & $\begin{array}{l}177 \\
(480)\end{array}$ & $\begin{array}{l}0 \\
(0-1,750)\end{array}$ & $\begin{array}{l}1,211 \\
(2,061)\end{array}$ & $\begin{array}{l}107 \\
(0-369)\end{array}$ & $\begin{array}{l}402 \\
(759)\end{array}$ & $\begin{array}{l}983 \\
(0-3,351)\end{array}$ & $\begin{array}{l}2,411 \\
(3,598)\end{array}$ \\
\hline \multicolumn{9}{|l|}{ By cancer type } \\
\hline $\begin{array}{l}\text { Acute lymphoblastic } \\
\text { leukemia }\end{array}$ & $\begin{array}{l}0 \\
(0-0)\end{array}$ & $\begin{array}{l}179 \\
(440)\end{array}$ & $\begin{array}{l}2,930 \\
(0-6,395)\end{array}$ & $\begin{array}{l}3,815 \\
(3,738)\end{array}$ & $\begin{array}{l}525 \\
(128-1,217)\end{array}$ & $\begin{array}{l}879 \\
(1,122)\end{array}$ & $\begin{array}{l}5,467 \\
(1,854-10,206)\end{array}$ & $\begin{array}{l}6,879 \\
(6,320)\end{array}$ \\
\hline Brain & $\begin{array}{l}0 \\
(0-0)\end{array}$ & $\begin{array}{l}249 \\
(597)\end{array}$ & $\begin{array}{l}1,548 \\
(0-3,881)\end{array}$ & $\begin{array}{l}2,454 \\
(2,981)\end{array}$ & $\begin{array}{l}181 \\
(0-818)\end{array}$ & $\begin{array}{l}643 \\
(1,071)\end{array}$ & $\begin{array}{l}2,406 \\
(622-5,382)\end{array}$ & $\begin{array}{l}4,020 \\
(4,820)\end{array}$ \\
\hline Hodgkin lymphoma & $\begin{array}{l}0 \\
(0-0)\end{array}$ & $\begin{array}{l}165 \\
(397)\end{array}$ & $\begin{array}{l}610 \\
(0-2,072)\end{array}$ & $\begin{array}{l}1,462 \\
(2,119)\end{array}$ & $\begin{array}{l}54 \\
(0-255)\end{array}$ & $\begin{array}{l}227 \\
(467)\end{array}$ & $\begin{array}{l}1,345 \\
(0-3,403)\end{array}$ & $\begin{array}{l}2,337 \\
(3,382)\end{array}$ \\
\hline $\begin{array}{l}\text { Non-Hodgkin } \\
\text { lymphoma }\end{array}$ & $\begin{array}{l}0 \\
(0-0)\end{array}$ & $\begin{array}{l}268 \\
(655)\end{array}$ & $\begin{array}{l}1,824 \\
(0-4,251)\end{array}$ & $\begin{array}{l}2,518 \\
(2,883)\end{array}$ & $\begin{array}{l}128 \\
(0-512)\end{array}$ & $\begin{array}{l}467 \\
(831)\end{array}$ & $\begin{array}{l}2,846 \\
(418-6,173)\end{array}$ & $\begin{array}{l}4,043 \\
(4,380)\end{array}$ \\
\hline Bone & $\begin{array}{l}0 \\
(0-0)\end{array}$ & $\begin{array}{l}119 \\
(363)\end{array}$ & $\begin{array}{l}533 \\
(0-2,930)\end{array}$ & $\begin{array}{l}1,902 \\
(2,815)\end{array}$ & $\begin{array}{l}117 \\
(0-453)\end{array}$ & $\begin{array}{l}475 \\
(867)\end{array}$ & $\begin{array}{l}1,446 \\
(411-4,183)\end{array}$ & $\begin{array}{l}3,251 \\
(4,363)\end{array}$ \\
\hline $\begin{array}{l}\text { Acute myeloid } \\
\text { leukemia }\end{array}$ & $\begin{array}{l}0 \\
(0-0)\end{array}$ & $\begin{array}{l}223 \\
(602)\end{array}$ & $\begin{array}{l}2,837 \\
(0-5,251)\end{array}$ & $\begin{array}{l}3,170 \\
(3,085)\end{array}$ & $\begin{array}{l}338 \\
(0-1,002)\end{array}$ & $\begin{array}{l}713 \\
(972)\end{array}$ & $\begin{array}{l}3,739 \\
(1,062-7,796)\end{array}$ & $\begin{array}{l}5,472 \\
(5,553)\end{array}$ \\
\hline $\begin{array}{l}\text { Connective tissue, } \\
\text { peripheral nerves }\end{array}$ & $\begin{array}{l}0 \\
(0-0)\end{array}$ & $\begin{array}{l}245 \\
(792)\end{array}$ & $\begin{array}{l}817 \\
(0-3,414)\end{array}$ & $\begin{array}{l}2,385 \\
(3,550)\end{array}$ & $\begin{array}{l}150 \\
(0-611)\end{array}$ & $\begin{array}{l}562 \\
(1,047)\end{array}$ & $\begin{array}{l}2,333 \\
(214-6,021)\end{array}$ & $\begin{array}{l}3,948 \\
(4,883)\end{array}$ \\
\hline Kidney & $\begin{array}{l}0 \\
(0-0)\end{array}$ & $\begin{array}{l}220 \\
(579)\end{array}$ & $\begin{array}{l}2,472 \\
(0-4,865)\end{array}$ & $\begin{array}{l}2,994 \\
(3,239)\end{array}$ & $\begin{array}{l}167 \\
(0-507)\end{array}$ & $\begin{array}{l}426 \\
(748)\end{array}$ & $\begin{array}{l}3,538 \\
(801-6,414)\end{array}$ & $\begin{array}{l}4,395 \\
(4,352)\end{array}$ \\
\hline $\begin{array}{l}\text { Other endocrine } \\
\text { glands }\end{array}$ & $\begin{array}{l}0 \\
(0-710)\end{array}$ & $\begin{array}{l}393 \\
(679)\end{array}$ & $\begin{array}{l}3,228 \\
(0-6,411)\end{array}$ & $\begin{array}{l}3,995 \\
(3,921)\end{array}$ & $\begin{array}{l}475 \\
(36-1,410)\end{array}$ & $\begin{array}{l}948 \\
(1,408)\end{array}$ & $\begin{array}{l}5,176 \\
(1,829-9,721)\end{array}$ & $\begin{array}{l}6,307 \\
(5,424)\end{array}$ \\
\hline Melanoma of skin & $\begin{array}{l}0 \\
(0-0)\end{array}$ & $\begin{array}{l}85 \\
(308)\end{array}$ & $\begin{array}{l}0 \\
(0-0)\end{array}$ & $\begin{array}{l}205 \\
(562)\end{array}$ & $\begin{array}{l}0 \\
(0-205)\end{array}$ & $\begin{array}{l}292 \\
(579)\end{array}$ & $\begin{array}{l}249 \\
(0-1,133)\end{array}$ & $\begin{array}{l}1,304 \\
(2,447)\end{array}$ \\
\hline Colon & $\begin{array}{l}0 \\
(0-411)\end{array}$ & $\begin{array}{l}341 \\
(724)\end{array}$ & $\begin{array}{l}983 \\
(0-2,079)\end{array}$ & $\begin{array}{l}1,337 \\
(1,390)\end{array}$ & $\begin{array}{l}103 \\
(0-436)\end{array}$ & $\begin{array}{l}316 \\
(456)\end{array}$ & $\begin{array}{l}1,745 \\
(510-4,271)\end{array}$ & $\begin{array}{l}2,563 \\
(2,661)\end{array}$ \\
\hline Thyroid & $\begin{array}{l}0 \\
(0-0)\end{array}$ & $\begin{array}{l}150 \\
(401)\end{array}$ & $\begin{array}{l}0 \\
(0-142)\end{array}$ & $\begin{array}{l}300 \\
(908)\end{array}$ & $\begin{array}{l}0 \\
(0-176)\end{array}$ & $\begin{array}{l}166 \\
(404)\end{array}$ & $\begin{array}{l}371 \\
(0-817)\end{array}$ & $\begin{array}{l}962 \\
(2,308)\end{array}$ \\
\hline Testis & $\begin{array}{l}0 \\
(0-0)\end{array}$ & $\begin{array}{l}97 \\
(296)\end{array}$ & $\begin{array}{l}0 \\
(0-1,317)\end{array}$ & $\begin{array}{l}1,057 \\
(2,250)\end{array}$ & $\begin{array}{l}103 \\
(0-263)\end{array}$ & $\begin{array}{l}366 \\
(788)\end{array}$ & $\begin{array}{l}787 \\
(0-2,578)\end{array}$ & $\begin{array}{l}2,062 \\
(2,992)\end{array}$ \\
\hline $\begin{array}{l}\text { Central nervous } \\
\text { system }\end{array}$ & $\begin{array}{l}0 \\
(0-0)\end{array}$ & $\begin{array}{l}214 \\
(562)\end{array}$ & $\begin{array}{l}0 \\
(0-2,118)\end{array}$ & $\begin{array}{l}1,370 \\
(2,068)\end{array}$ & $\begin{array}{l}258 \\
(0-718)\end{array}$ & $\begin{array}{l}516 \\
(729)\end{array}$ & $\begin{array}{l}1,351 \\
(379-5,761)\end{array}$ & $\begin{array}{l}3,261 \\
(3,913)\end{array}$ \\
\hline $\begin{array}{l}\text { III-defined and } \\
\text { unspecified site }\end{array}$ & $\begin{array}{l}0 \\
(0-0)\end{array}$ & $\begin{array}{l}228 \\
(512)\end{array}$ & $\begin{array}{l}509 \\
(0-3,806)\end{array}$ & $\begin{array}{l}2,334 \\
(3,243)\end{array}$ & $\begin{array}{l}246 \\
(103-953)\end{array}$ & $\begin{array}{l}586 \\
(825)\end{array}$ & $\begin{array}{l}1,948 \\
(625-6,356)\end{array}$ & $\begin{array}{l}4,385 \\
(5,132)\end{array}$ \\
\hline Eye & $\begin{array}{l}0 \\
(0-0)\end{array}$ & $\begin{array}{l}153 \\
(388)\end{array}$ & $\begin{array}{l}512 \\
(0-2,180)\end{array}$ & $\begin{array}{l}1,473 \\
(1,982)\end{array}$ & $\begin{array}{l}145 \\
(0-357)\end{array}$ & $\begin{array}{l}350 \\
(556)\end{array}$ & $\begin{array}{l}1,675 \\
(411-4,315)\end{array}$ & $\begin{array}{l}2,818 \\
(3,566)\end{array}$ \\
\hline $\begin{array}{l}\text { Other lymphatic, } \\
\text { hematopoietic }\end{array}$ & $\begin{array}{l}0 \\
(0-0)\end{array}$ & $\begin{array}{l}406 \\
(1,021)\end{array}$ & $\begin{array}{l}724 \\
(0-1,741)\end{array}$ & $\begin{array}{l}1,663 \\
(2,906)\end{array}$ & $\begin{array}{l}244 \\
(54-622)\end{array}$ & $\begin{array}{l}442 \\
(515)\end{array}$ & $\begin{array}{l}1,741 \\
(975-4,458)\end{array}$ & $\begin{array}{l}3,361 \\
(3,931)\end{array}$ \\
\hline Liver & $\begin{array}{l}0 \\
(0-448)\end{array}$ & $\begin{array}{l}256 \\
(482) \\
\end{array}$ & $\begin{array}{l}3,352 \\
(0-5,665)\end{array}$ & $\begin{array}{l}3,371 \\
(3,178)\end{array}$ & $\begin{array}{l}360 \\
(0-663)\end{array}$ & $\begin{array}{l}711 \\
(1,414)\end{array}$ & $\begin{array}{l}3,352 \\
(1,470-8,591)\end{array}$ & $\begin{array}{l}5,832 \\
(7,009) \\
\end{array}$ \\
\hline Ovary & $\begin{array}{l}0 \\
(0-0)\end{array}$ & $\begin{array}{l}120 \\
(322)\end{array}$ & $\begin{array}{l}389 \\
(0-1,264)\end{array}$ & $\begin{array}{l}1,444 \\
(3,311)\end{array}$ & $\begin{array}{l}0 \\
(0-225)\end{array}$ & $\begin{array}{l}230 \\
(601)\end{array}$ & $\begin{array}{l}960 \\
(0-2,296)\end{array}$ & $\begin{array}{l}2,364 \\
(5,488)\end{array}$ \\
\hline $\begin{array}{l}\text { Other myeloid } \\
\text { leukemia }\end{array}$ & $\begin{array}{l}0 \\
(0-0)\end{array}$ & $\begin{array}{l}166 \\
(506)\end{array}$ & $\begin{array}{l}1,625 \\
(0-2,651)\end{array}$ & $\begin{array}{l}1,568 \\
(1,406)\end{array}$ & $\begin{array}{l}185 \\
(0-773)\end{array}$ & $\begin{array}{l}565 \\
(806)\end{array}$ & $\begin{array}{l}2,911 \\
(453-4,769)\end{array}$ & $\begin{array}{l}3,558 \\
(3,608)\end{array}$ \\
\hline $\begin{array}{l}\text { Other thoracic } \\
\text { organs }\end{array}$ & $\begin{array}{l}0 \\
(0-503)\end{array}$ & $\begin{array}{l}499 \\
(1,082)\end{array}$ & $\begin{array}{l}2,546 \\
(0-6,202)\end{array}$ & $\begin{array}{l}3,677 \\
(3,949)\end{array}$ & $\begin{array}{l}139 \\
(71-401)\end{array}$ & $\begin{array}{l}393 \\
(580)\end{array}$ & $\begin{array}{l}3,356 \\
(710-7,857)\end{array}$ & $\begin{array}{l}4,959 \\
(5,072)\end{array}$ \\
\hline $\begin{array}{l}\text { All other cancer } \\
\text { types }\end{array}$ & $\begin{array}{l}0 \\
(0-453)\end{array}$ & $\begin{array}{l}324 \\
(750)\end{array}$ & $\begin{array}{l}0 \\
(0-2,900)\end{array}$ & $\begin{array}{l}1,843 \\
(2,823)\end{array}$ & $\begin{array}{l}110 \\
(0-430)\end{array}$ & $\begin{array}{l}447 \\
(869)\end{array}$ & $\begin{array}{l}1,443 \\
(0-5,175)\end{array}$ & $\begin{array}{l}3,329 \\
(4,694)\end{array}$ \\
\hline
\end{tabular}

Abbreviation: IQR, interquartile range. 
eTable 8. Cost of Cancer-Related Hospital Admissions and ED Presentations in Australian Dollars

\begin{tabular}{|c|c|c|}
\hline & $\begin{array}{l}\text { Median Cost of } \\
\text { Hospital + ED (IQR), A\$ }\end{array}$ & $\begin{array}{l}\text { Average Cost of } \\
\text { Hospital + ED (SD), AS }\end{array}$ \\
\hline \multicolumn{3}{|l|}{ Age at diagnosis } \\
\hline $0-14$ y & $123,405(46,407-221,804)$ & $167,506(165,517)$ \\
\hline $15-17 y$ & $32,309(8,676-125,958)$ & $92,678(136,890)$ \\
\hline \multicolumn{3}{|l|}{ By cancer type } \\
\hline Acute lymphoblastic leukemia & $161,385(106,321-283,967)$ & $222,939(179,406)$ \\
\hline Brain & $108,419(35,090-208,907)$ & $153,968(163,358)$ \\
\hline Hodgkin lymphoma & $24,052(9,610-51,336)$ & $41,058(53,213)$ \\
\hline Non-Hodgkin lymphoma & $101,724(44,961-189,568)$ & $141,319(150,804)$ \\
\hline Bone & $174,408(80,124-258,400)$ & $180,808(133,549)$ \\
\hline Acute myeloid leukemia & $242,376(145,155-362,766)$ & $273,261(178,849)$ \\
\hline Connective tissue, peripheral nerves & $90,200(36,116-180,537)$ & $132,173(135,001)$ \\
\hline Kidney & $75,388(40,506-140,921)$ & $112,915(139,500)$ \\
\hline Other endocrine glands & $205,872(82,092-323,625)$ & $217,873(161,241)$ \\
\hline Melanoma of skin & $2,611(742-7,188)$ & $9,327(22,414)$ \\
\hline Colon & $11,030(5,546-17,962)$ & $16,675(21,097)$ \\
\hline Thyroid & $22,979(17,219-32,309)$ & $31,002(40,869)$ \\
\hline Testis & $20,425(5,180-71,872)$ & $52,891(78,092)$ \\
\hline Central nervous system & $59,722(25,244-130,620)$ & $92,915(95,019)$ \\
\hline III-defined and unspecified site & $124,685(43,115-247,829)$ & $159,540(138,232)$ \\
\hline Eye & $58,650(36,031-111,528)$ & $89,180(88,365)$ \\
\hline Other lymphatic, hematopoietic & $18,872(10,205-106,665)$ & $90,369(160,847)$ \\
\hline Liver & $107,551(57,391-164,642)$ & $141,732(132,501)$ \\
\hline Ovary & $28,750(14,687-68,287)$ & $50,866(58,864)$ \\
\hline Other myeloid leukemia & $60,942(17,682-238,169)$ & $134,842(145,448)$ \\
\hline Other thoracic organs & $136,402(83,815-254,510)$ & $172,239(124,776)$ \\
\hline All other cancer types & $41,813(13,307-168,170)$ & $128,171(174,662)$ \\
\hline Total sum & $447,802,210$ & \\
\hline
\end{tabular}

Total costs of all cancer-related hospital admissions and emergency department presentations were $\mathrm{A} \$ 448$ million for the 5 -year period after cancer diagnosis for all patients in New South Wales from 2001 through 2012. Costs are presented in 2018 Australian dollars.

Abbreviations: ED, emergency department; IQR, interquartile range. 


\begin{tabular}{|c|c|}
\hline Major Diagnostic Category & n (\%) \\
\hline \multicolumn{2}{|l|}{ Cancer-related admission } \\
\hline Neoplastic disorders (hematologic and solid neoplasms) & $21,096(43.90)$ \\
\hline Diseases and disorders of the musculoskeletal system and connective tissue & $4,233(8.81)$ \\
\hline Infectious and parasitic diseases & $3,681(7.66)$ \\
\hline Diseases and disorders of the nervous system & $3,504(7.29)$ \\
\hline Diseases and disorders of the blood and blood-forming organs and immunologic disorders & $3,471(7.22)$ \\
\hline Diseases and disorders of the kidney and urinary tract & $1,687(3.51)$ \\
\hline Endocrine, nutritional and metabolic diseases and disorders & $1,526(3.18)$ \\
\hline Diseases and disorders of the digestive system & $1,512(3.15)$ \\
\hline Factors influencing health status and other contacts with health services & $1,499(3.12)$ \\
\hline Diseases and disorders of the ear, nose, mouth and throat & $1,187(2.47)$ \\
\hline Diseases and disorders of the respiratory system & $1,133(2.36)$ \\
\hline Diseases and disorders of the eye & $1,075(2.24)$ \\
\hline Diseases and disorders of the circulatory system & $597(1.24)$ \\
\hline Diseases and disorders of the hepatobiliary system and pancreas & $539(1.12)$ \\
\hline Diseases and disorders of the skin, subcutaneous tissue and breast & $416(0.87)$ \\
\hline Diseases and disorders of the male reproductive system & $343(0.71)$ \\
\hline Diseases and disorders of the female reproductive system & $306(0.64)$ \\
\hline Injuries, poisoning and toxic effect of drugs & $142(0.30)$ \\
\hline All other MDCs & $113(0.23)$ \\
\hline \multicolumn{2}{|l|}{ Admission records without MDCs $=30$} \\
\hline \multicolumn{2}{|l|}{ Non-cancer-related admission } \\
\hline Factors influencing health status and other contacts with health services & $1,284(20.46)$ \\
\hline Diseases and disorders of the kidney and urinary tract & 715 (11.39) \\
\hline Diseases and disorders of the ear, nose, mouth, and throat & $568(9.05)$ \\
\hline Diseases and disorders of the blood and blood-forming organs and immunologic disorders & $528(8.41)$ \\
\hline Diseases and disorders of the digestive system & $517(8.24)$ \\
\hline Infectious and parasitic diseases & $392(6.25)$ \\
\hline Diseases and disorders of the musculoskeletal system and connective tissue & $377(6.01)$ \\
\hline Diseases and disorders of the nervous system & $324(5.16)$ \\
\hline Endocrine, nutritional, and metabolic diseases and disorders & $322(5.13)$ \\
\hline Diseases and disorders of the respiratory system & $311(4.96)$ \\
\hline Diseases and disorders of the skin, subcutaneous tissue, and breast & $205(3.27)$ \\
\hline Diseases and disorders of the circulatory system & $162(2.58)$ \\
\hline Injuries, poisoning, and toxic effect of drugs & $122(1.94)$ \\
\hline All other MDCs & $449(7.16)$ \\
\hline Admission records without $\mathrm{MDC}=33$ & \\
\hline
\end{tabular}

Abbreviation: MDC, major diagnostic category. 


\begin{tabular}{|c|c|c|c|c|c|c|}
\hline \multirow[b]{2}{*}{ Parameter } & \multicolumn{2}{|c|}{ No. of Hospital Admissions } & \multicolumn{2}{|c|}{ Hospital LoS } & \multicolumn{2}{|c|}{ Cost of Hospital Admission } \\
\hline & Estimate & $P$ Value & Estimate & $P$ Value & Estimate & $P$ Value \\
\hline Intercept & 3.57 & .8799 & 0.00 & .3125 & 0.00 & .324 \\
\hline Age at diagnosis & 0.97 & $<.0001$ & 0.98 & $<.0001$ & 0.98 & $<.0001$ \\
\hline Sex & & .038 & & .1078 & & .1538 \\
\hline Female & Ref & & Ref & & Ref & \\
\hline Male & 1.06 & .0377 & 1.06 & .1074 & 1.05 & .1534 \\
\hline \multicolumn{2}{|c|}{ Highest degree of spread within 4 mo of diagnosis } & $<.0001$ & & $<.0001$ & & $<.0001$ \\
\hline Localized & Ref & & Ref & & Ref & \\
\hline Regionalized & 1.23 & .0002 & 1.68 & $<.0001$ & 1.65 & $<.0001$ \\
\hline Distant metastases & 1.61 & $<.0001$ & 2.21 & $<.0001$ & 2.03 & $<.0001$ \\
\hline Hematologic cancer/unknown spread & 1.46 & .0115 & 2.79 & $<.0001$ & 2.69 & $<.0001$ \\
\hline Cancer type & & $<.0001$ & & $<.0001$ & & $<.0001$ \\
\hline Acute lymphoblastic leukemia & Ref & & Ref & & Ref & \\
\hline Brain & 0.73 & .0482 & 1.82 & .0017 & 2.22 & $<.0001$ \\
\hline Hodgkin lymphoma & 0.38 & $<.0001$ & 0.25 & $<.0001$ & 0.27 & $<.0001$ \\
\hline Non-Hodgkin lymphoma & 0.61 & $<.0001$ & 0.86 & .043 & 0.93 & .305 \\
\hline Bone & 1.34 & .0722 & 2.68 & $<.0001$ & 2.66 & $<.0001$ \\
\hline Acute myeloid leukemia & 0.63 & $<.0001$ & 1.45 & $<.0001$ & 1.49 & $<.0001$ \\
\hline Connective tissue, peripheral nerves & 0.83 & .2467 & 1.30 & .193 & 1.32 & .1413 \\
\hline Kidney & 0.69 & .026 & 1.25 & .2697 & 1.22 & .2835 \\
\hline Other endocrine glands & 0.78 & .1396 & 1.90 & .0017 & 1.89 & .001 \\
\hline Melanoma of skin & 0.10 & $<.0001$ & 0.07 & $<.0001$ & 0.12 & $<.0001$ \\
\hline Colon & 0.13 & $<.0001$ & 0.32 & $<.0001$ & 0.44 & .0004 \\
\hline Thyroid & 0.23 & $<.0001$ & 0.23 & $<.0001$ & 0.43 & $<.0001$ \\
\hline Testis & 0.58 & .0026 & 0.63 & .0332 & 0.64 & .0271 \\
\hline Central nervous system & 0.46 & $<.0001$ & 0.79 & .2814 & 1.00 & .9899 \\
\hline III-defined and unspecified site & 0.51 & $<.0001$ & 0.73 & .0113 & 0.78 & .0266 \\
\hline Eye & 0.63 & .011 & 0.58 & .0135 & 0.77 & .2136 \\
\hline Other lymphatic, hematopoietic & 0.28 & $<.0001$ & 0.32 & $<.0001$ & 0.36 & $<.0001$ \\
\hline Liver & 0.80 & .2584 & 1.80 & .0115 & 1.78 & .0085 \\
\hline Ovary & 0.46 & $<.0001$ & 0.77 & .2772 & 0.63 & .0373 \\
\hline Other myeloid leukemia & 0.22 & $<.0001$ & 0.60 & .0033 & 0.77 & .1246 \\
\hline Other thoracic organs & 0.80 & .31 & 1.29 & .3487 & 1.38 & .2064 \\
\hline All other cancer types & 0.64 & .0003 & 1.04 & .798 & 1.15 & .3202 \\
\hline Number of primary cancer sites & & .8569 & & .0692 & & .965 \\
\hline 1 & Ref & & Ref & & Ref & \\
\hline$\geq 2$ & 1.02 & .8661 & 1.31 & .0782 & 1.01 & .965 \\
\hline ARIA+ (patient's geographic remoteness) & & .0794 & & .0448 & & .2338 \\
\hline Major cities & Ref & & Ref & & Ref & \\
\hline Inner regional & 0.99 & .8661 & 0.89 & .0085 & 0.93 & .109 \\
\hline Outer regional & 0.98 & .6826 & 0.90 & .1399 & 1.00 & .9706 \\
\hline Remote and very remote & 1.60 & .0157 & 1.06 & .8063 & 1.35 & .2395 \\
\hline
\end{tabular}

(continued on next page) 


\begin{tabular}{|c|c|c|c|c|c|c|}
\hline \multirow[b]{2}{*}{ Parameter } & \multicolumn{2}{|c|}{ No. of Hospital Admissions } & \multicolumn{2}{|c|}{ Hospital LoS } & \multicolumn{2}{|c|}{ Cost of Hospital Admission } \\
\hline & Estimate & $P$ Value & Estimate & $P$ Value & Estimate & $P$ Value \\
\hline SEIFA-IRSD & & .0004 & & .0684 & & .126 \\
\hline Quintile 1 (least disadvantaged) & Ref & & Ref & & Ref & \\
\hline Quintile 2 & 0.89 & .0095 & 1.04 & .4969 & 1.08 & .1309 \\
\hline Quintile 3 & 0.99 & .7523 & 1.00 & .9592 & 1.07 & .2195 \\
\hline Quintile 4 & 1.01 & .865 & 1.09 & .1268 & 1.09 & .0907 \\
\hline Quintile 5 (most disadvantaged) & 0.87 & .0015 & 1.14 & .0171 & 1.14 & .008 \\
\hline Diagnosis year & 1.00 & .857 & 1.01 & .1818 & 1.01 & .0335 \\
\hline
\end{tabular}

Abbreviations: ARIA, Accessibility/Remoteness Index of Australia; IRSD, Index of Relative Socio-economic Disadvantage; LoS, length of stay; SEIFA, Socio-Economic Indexes for Areas. 